\title{
Sample of minor merger of galaxies (Research Note)
}

\section{Modelling HII region properties ${ }^{\star}$}

\author{
D. L. Ferreiro ${ }^{1, \star \star}$, M. G. Pastoriza ${ }^{2}$, and M. Rickes ${ }^{2}$ \\ 1 IATE, Observatorio Astronómico, Universidad Nacional de Córdoba, Laprida 854, 5000 Córdoba, Argentina \\ e-mail: diegof@mail.oac.uncor.edu \\ 2 Departamento de Astronomia, IF-UFRGS, CP 15051, CEP 91501-970, Porto Alegre, RS, Brazil \\ e-mail: [miriani.pastoriza;mauro]@ufrgs.br
}

Received 21 July 2006 / Accepted 14 January 2008

ABSTRACT

\begin{abstract}
Context. Studies of interacting galaxies have shown that dwarf galaxies may be produced in tidal tails during collisions. Numerical simulations suggest that the total detachment of the tidal dwarf requires the companion's mass to be comparable to or higher than that of the parent galaxy. This would imply that minor mergers (interaction between unequal mass galaxies) might not form tidal dwarf galaxies (TDGs)

Aims. The objectives of the paper is to analyse the main properties of a sample of $116 \mathrm{HII}$ regions previously selected from $\mathrm{H} \alpha$ images of 11 minor mergers of galaxies and discuss the nature of these regions comparing their properties with those observed in HII regions in normal and isolated galaxies and TDG candidates.

Methods. Individual parameters such as: blue absolute magnitude, $M_{B}, \mathrm{H} \alpha$ luminosity, $\mathcal{L}(\mathrm{H} \alpha)$, star formation rate, $S F R(\mathrm{H} \alpha)$ and mass, $M / M_{\odot}$ of these regions were derived and compared with those of the HII regions in normal isolated galaxies, as well as in tidal dwarf candidates.

Results. A full $62 \%$ of the HII regions are brighter than $M_{B} \leq-15$ mag, which is an upper limit for giant HII complexes observed in the spiral arms of $\mathrm{Sa}$ and $\mathrm{Sb}$ galaxies. Five regions are as bright as the tidal dwarf candidates in the tidal tail of Arp 105. It was also found that 43 regions have $\mathrm{H} \alpha$ luminosities brighter than $10^{40} \mathrm{erg} \mathrm{s}^{-1}$, which is the upper luminosity limit for HII regions in normal Sc galaxies. The estimated stellar masses of $32 \mathrm{HII}$ regions are $1 \times 10^{6}<M / M_{\odot}<3 \times 10^{7}$. These values are comparable to those of young massive clusters (YMC).

Conclusions. In our sample we have detected $9 \mathrm{HII}$ regions presenting $\mathrm{H} \alpha$ luminosities which correspond to star formation rates higher than those found in normal HII regions in spiral galaxies. These regions are associated with very massive clusters $\left(1 \times 10^{8} M_{\odot}\right)$ typical of TDGs.
\end{abstract}

Key words. galaxies: interactions - galaxies: starburst - galaxies: photometry - publications, bibliography

\section{Introduction}

Spatially resolved spectrophotometry observations of spiral galaxies indicate that the strong trend of star formation activity along the Hubble sequence is due to increases in both the luminosity of the individual HII region and the number of starforming events per unit area (Kennicutt et al. 1989). There is an order of magnitude gap between the luminosity of the brightest $\mathrm{HII}$ region in Sa and Sc-Irr galaxies. Although it is commonly assumed that this difference is due to changes in the characteristic number of stars in the star-formin region. Bresolin \& Kennicutt (1997) found that the properties of star forming region are virtually the same in two samples of late-type and early-type spirals, except for their luminosities. The $\mathrm{Sa}$ and early $\mathrm{Sb}$ spiral have no HII region brighter than $\mathcal{L}(\mathrm{H} \alpha)=10^{39} \mathrm{erg} \mathrm{s}^{-1}$, while in late-type spirals and irregulars the most luminous HII region are brighter than $\mathcal{L}(\mathrm{H} \alpha)=10^{40} \mathrm{erg} \mathrm{s}^{-1}$. In particular, they did not find significant difference in $E W(\mathrm{H} \alpha)$ values and colours between the two

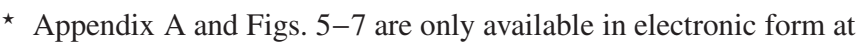
http://www . aanda.org

$\star \star$ Visiting Astronomer at the Cerro Tololo Iter-American Observatory (CTIO).
}

samples. However, the results by Hameed \& Devereux (2005) do not completely agree with the $\mathrm{H} \alpha$ study of Kennicutt \& Kent (1983). These authors find a significant fraction of early-type spiral galaxies with unexpectedly high values in $E W(\mathrm{H} \alpha)$ and with a "super-giant" HII region $\left(\mathcal{L}(\mathrm{H} \alpha) \geq 10^{39} \mathrm{erg} \mathrm{s}^{-1}\right)$. They suggest that recent minor mergers or past interactions are responsible for the high elevated levels of $\mathrm{H} \alpha$ emission and for the presence of giant HII regions in these galaxies.

Beckman et al. (1998) presented evidence that favours the hypothesis that HII regions with luminosities greater than a critical value of $10^{38.6} \mathrm{erg} \mathrm{s}^{-1}$ in disk galaxies are density-bounded and that the escaping Lyman continuum photons from them are the principal ionizing agent of the diffuse ISM in disk galaxies. There is a low-level background of diffuse $\mathrm{H} \alpha$ that seems unimportant, but it has been shown to comprise some $50 \%$ of the total $\mathrm{H} \alpha$ luminosity of a typical late-type galaxy (Zurita et al. 2000), occupying over $80 \%$ of the disk area (Beckman et al. 2002). Although a fraction of the ionization of the diffuse ionized component of the ISM may well be caused by shock or turbulence (Hoopers \& Walterbos 2003), the major fraction of this ionization must probably come from the OB stars in HII regions (Giammanco et al. 2005). 
Using the observed mass, Duc et al. (2004) classify the starforming regions in galaxies into the following categories:

\section{- super stellar clusters :}

These regions have typical masses of $10^{5} M_{\odot}$ and appear on ground-based optical images as compact stellar condensations. They are located either in the central regions of mergers (Holtzman et al. 1992; Whitmore et al. 1999; Zepf et al. 1999), in tidal bridges, or along extended tidal tails (Gallagher et al. 2001; Knierman et al. 2003; Saviane et al. 2004) and may be enshrouded by dust (Gilbert et al. 2000).

- young massive clusters (YMC):

These objects have been identified in high-resolution HST images (de Grijs et al. 2003; Tran et al. 2003). Their blue luminosity, inferred mass, and size are such that some of them may evolve into Globular Clusters with typical masses of $10^{6} M_{\odot}$ (Schweizer et al. 1996). Some $10^{7} M_{\odot} \mathrm{HI}$ fragments discovered around mergers could be their gaseous progenitors (English et al. 2003).

- giant HII complexes $(G H I I C)$.

Star-forming regions with luminosities exceeding the giant HII regions found in isolated spiral disks were discovered in long optical tidal tails (Weilbacher et al. 2003; López-Sánchez et al. 2004). "Intergalactic emission line region", characterised by a very low underlying old stellar content and by their compact aspect, were detected in several clusters of galaxies. Their optical spectra are typical of starforming HII regions and their rather high oxygen abundances indicate that they are formed of pre-enriched gas probably stripped from parent colliding galaxies (Gerhard et al. 2002; Cortese et al. 2004; Ryan-Weber et al. 2004; Mendes de Oliveira et al. 2004).

- tidal dwarf galaxies candidates (TDGs):

The TDG candidates are objects with the apparent masses and sizes of dwarf galaxies. They contain large quantities of gas in atomic, molecular, and ionized form and have luminous masses typically of $10^{9} M_{\odot}$ (Braine et al. 2001). Regions with luminosities brighter than $M_{B}<-15$ mag have been found. They are most often observed at or near the tip of long tidal tails (Hunter et al. 2000; Weilbacher et al. 2004). For example, Iglesias-Páramo \& Vílchez (2001) searched for star-forming objects belonging to tidal tails in a sample of deep $\mathrm{H} \alpha$ images for 16 compact groups of galaxies. A total of 36 objects with $\mathrm{H} \alpha$ luminosities higher than $10^{38} \mathrm{erg} \mathrm{s}^{-1}$ has been detected in five groups. The fraction of the total $\mathrm{H} \alpha$ flux corresponding to tidal objects is typically under $5 \%$ for most of the objects. Out of these 36 objects, nine starforming TDG candidates were identified on the bases of their projected distance to the nuclei of the parent galaxies and of their total $\mathrm{H} \alpha$ luminosities. Indeed an apparent accumulation of tidal material could be artificial. In 3D space, tidal tails are curved. Seen edge-on, they appear as linear structures and may show at their tip fake mass concentrations caused by material projected along the line of sight (Hibbard \& Barnes 2004; Mihos 2004). These objects around mergers all contain stars with ages, derived from their colours or spectroscopic properties, which are comparable to or less than the dynamical age of the collision between the parent galaxies. They were thus most probably formed during the merging process.

In a previous paper, we studied and determined basic photometric parameters of the HII regions of a sample of eleven minor mergers ${ }^{1}$ of galaxies (Ferreiro \& Pastoriza 2004, hereafter FP2004). We found that the HII regions of the sample have an average age of $(6.3 \pm 0.7) \mathrm{Myr}$, luminosity between $38.6<\log (\mathcal{L}(\mathrm{H} \alpha+\mathrm{NII}))<41.7$ and that the luminosity function of the whole sample fits a power law of index $\alpha=-1.33$ showing an excess of very luminous objects compared with normal galaxies.

The goal of this paper is to study the properties of the HII regions of the sample of minor mergers according to the individual parameters: blue absolute magnitude $M_{B}, \mathrm{H} \alpha$ luminosity $\mathcal{L}(\mathrm{H} \alpha)$, star formation rate $\operatorname{SFR}(\mathrm{H} \alpha)$, and their stellar mass $M$. These parameters allow us to discuss the nature of the star-forming regions comparison to the others HII regions in normal and isolated galaxies and TDG candidates. The paper is organised as follows. In Sect. 2 we briefly describe the sample, the observations, and the data reduction. In Sect. 3 we describe the use of the synthesis model of Leitherer et al. (1999; Starburst99) used to estimate the age and mass of the HII regions. In Sect. 4 we describe the nature of the ionizing cluster. Section A describes the particular properties of the HII regions in the individual galaxies. In Sect. 5 we present the final remarks and conclusions.

\section{Sample, observation, and data reduction}

The sample selection, observations, and data reduction were described in detail in FP2004. The pairs of galaxies were minor mergers selected from the Arp \& Madore (1987) catalogue. All images $^{2}$ were reduced and calibrated following the standard procedures of IRAF package. All the distance-dependent quantities were estimated by adopting $H_{0}=75 \mathrm{~km} \mathrm{~s}^{-1} \mathrm{Mpc}^{-1}$. A suitable $\mathrm{H} \alpha$ continuum image was subtracted for each $\mathrm{H} \alpha$ image. The HII region as identified as the region corresponding to maximum flux in the $\mathrm{H} \alpha$ maps, where the limiting criterion was to be larger than 5 sigma of the background. Besides, the size of the region has to be greater than the seeing $\left(1.3^{\prime \prime}\right)$. The HII region limit was measured using the area $(\mathcal{A})$ inside the isophote level that has an intensity value of $10 \%$ of the central intensity of the HII region. From this area, we calculated the equivalent radius as $r_{\mathrm{eq}}=(\mathcal{A} / \pi)^{0.5}$, and for each region we measured the luminosity and the equivalent width of $\mathrm{H} \alpha$. Using the same criterion, the HII region in the broad band was identified. The magnitudes and colours were measured by integrating the intensity pixels inside an area at the same positions in the $B, V$, and $I$ images, with the same form and size. We estimated the internal reddening for each galaxy from the line intensity ratio $\mathrm{H} \alpha / \mathrm{H} \beta$ observed in their central region by Pastoriza et al. (1999). In addition, we estimated the underlying disk emission using the luminosity profile. Since we know the distance from the centre of the galaxy to the HII region and had previously determined the luminosity profile of the disk, we were able to infer the value of magnitude per unit area of the underlying emission at that point. Based on that value, we estimated the background emission for the whole HII region area. The disk contribution to the HII region flux measured at the same position was found to be about $1 \%$ for most of the regions. The estimated values were inadequate compared with the photometric errors (approx 10\%). As described in FP2004, a total of $116 \mathrm{HII}$ regions were identified in the 11 minor merger pairs. In Tables A.1 and A.2 we present the star-forming regions, sorted according to increasing right ascension, with their main properties. Table A.1 lists identification, right ascension and

\footnotetext{
1 The condition for the minor merger is $M_{\text {(secondary) }} / M_{\text {(primary) }}<0.2$.

2 Image $B, V, I$ and $\mathrm{H} \alpha$ of the sample available at

http://www . aanda. org
} 
declination, absolute $M_{B}$ magnitude, integrated colours $(B-V)$ and $(V-I)$, and reddening $(E(B-V))$. We present in Table A.2 the $\mathrm{H} \alpha$ luminosity, $\log$ equivalent width $\left(\log (E W),\left[\operatorname{erg~s}^{-} 1\right]\right)$, age $([\mathrm{Myr}]), \operatorname{SFR}(\mathrm{H} \alpha)\left(\left[M_{\odot} \mathrm{yr}^{-1}\right]\right)$, and estimated mass $\left(\left[M_{\odot}\right]\right)$ of the observed HII region.

\section{Modelling the ionizing cluster}

\subsection{Star formation rate}

To estimate the star formation rate $(\operatorname{SFR}(\mathrm{H} \alpha))$ in each HII region, we determined the number of ionizing photons $Q(H)$ of the $\mathrm{H} \alpha$ luminosity listed in Table A. 2 corrected by the contribution of the [NII] $\lambda \lambda 6548,6584$ emission. We assumed the contribution to be $20 \%$ in all regions. This correction was derived from spectroscopic observations of galactic HII regions (Girardi et al. 1997). The number of ionizing photons was estimated from the Eq. (1) of Osterbrock (1989):

$Q(H)=\frac{L_{\mathrm{H} \alpha}}{h v_{\alpha}} \frac{\alpha_{B}\left(H^{0}, T\right)}{\alpha_{\mathrm{H} \alpha}\left(H^{0}, T\right)} 10^{c(\mathrm{H} \alpha)}$,

where $\alpha_{B}\left(H^{0}, T\right)$ is the coefficient of recombination added over all levels of energy, except that the fundamental one $\mathrm{H} \alpha\left(H^{0}, T\right)$ is the coefficient of recombination in $\alpha$ and the term $10^{c(\mathrm{H} \alpha)}$.

We calculated the $\operatorname{SFR}(\mathrm{H} \alpha)$ in the observed HII regions following Kennicutt (1998):

$\operatorname{SFR}(\mathrm{H} \alpha)=7.9 \times 10^{-42} \mathcal{L}(\mathrm{H} \alpha) \mathcal{M}_{\odot} \mathrm{yr}^{-\infty}$.

This equation was derived using the calibrations of Kennicutt et al. (1994) and Madau et al. (1998), assuming solar abundances and Salpeter's initial mass function (IMF) on a mass interval of $0.1 M_{\odot}<M_{\star}<100 M_{\odot}$. The $S F R$ values are listed in Table A.2.

Since we did not have the $\mathrm{H} \alpha$ calibration for all galaxies, we were able to determine the $S F R$ using the calibration between the number of ionizing photons $\left(Q\left(H_{0}\right)\right)$ and $S F R$. The number of ionizing photons is given by Starburst99 models (Leitherer et al. 1999, hereafter SB99), which depend on the mass and the age (see Sect. 3.2) of the observed HII regions. The following equation was used to calculate the $\operatorname{SFR}\left(Q\left(H_{0}\right)\right)$ in the observed HII regions (Kennicutt 1998):

$\operatorname{SFR}\left(Q\left(H_{0}\right)\right)=1.08 \times 10^{-53} Q\left(H_{0}\right) M_{\odot} \mathrm{yr}^{-1}$,

where $Q\left(H_{0}\right)$ is the number of ionizing photons by second. In Fig. 5 we show the percentage difference between $\operatorname{SFR}(\mathrm{H} \alpha)$ and $\left.\operatorname{SFR}\left(Q H_{0}\right)\right)$ relative to $\operatorname{SFR}(\mathrm{H} \alpha)$ for the regions with $\mathrm{H} \alpha$ calibration sorted by increasing right ascension. The new estimations agree with most of the observed HII regions. We found that the differences between $\operatorname{SFR}(\mathrm{H} \alpha)$ and $\left.\operatorname{SFR}\left(Q H_{0}\right)\right)$ is less than $30 \%$ for $56 \mathrm{HII}$ regions, whith is $\sim 60 \%$ of the HII regions with $\operatorname{SFR}(\mathrm{H} \alpha)$ measured. The same difference is greater than $50 \%$ for 12 HII regions. We include in Table A.2 the $\operatorname{SFR}\left(Q\left(H_{0}\right)\right)$ for the regions without $\mathrm{H} \alpha$ calibration.

\subsection{Modelling HII region properties}

To obtain more insight into internal properties that govern the star formation process in the observed HII regions, we used the SB99 code (Leitherer et al. 1999). We computed 13 evolutionary models for instantaneous bursts with different stellar masses ranging from $10^{5} M_{\odot}$ to $10^{9} M_{\odot}$. The following sub-sets were computed: $0.1,0.3,0.5,1,1.3,1.5,10,30,50,100,300,500$,

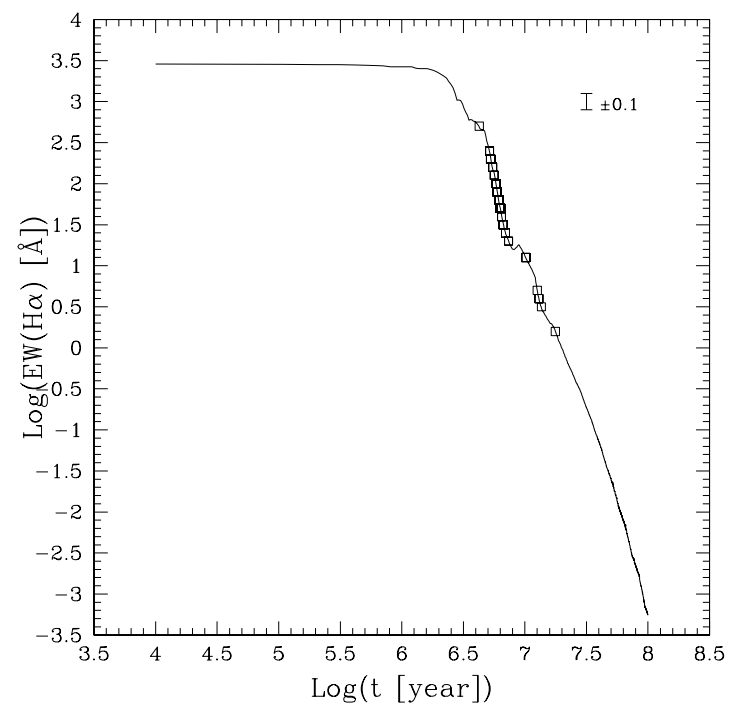

Fig. 1. Evolutionary track $E W$ for HII regions determined by the Starburst 99 model. Open squares correspond to the measured $E W(\mathrm{H} \alpha)$ for the observed HII regions. The upper right shows an uncertainty bar of $\log (E W(\mathrm{H} \alpha))=0.1 \AA$.

and 1000 in units of $10^{6} M_{\odot}$. For the remaining input parameters, default values were adopted. We chose Padove tracks with AGB stars, using theoretical wind models whose onset epoch of star formation was $10^{4}$ years, with a time step of $10^{5}$ years. The IMF power-law exponents were 1.3 and 2.3. The Pauldrach-Hilliers atmosphere model with solar abundance was used.

We estimated HII regions' ages through the observed equivalent widths of the $\mathrm{H} \alpha$ line $(E W(\mathrm{H} \alpha))$ and compared them with the ages of SB99 models. Note that the $E W$ of the HII regions is independent of the stellar cluster mass and does not depend on the internal reddening either. The evolutionary track for the $E W$ model is shown in Fig. 1. The open squares plotted over the model correspond to the $E W(\mathrm{H} \alpha)$ of the observed HII regions. It can be seen that ages are concentrated in the range $4.2 \times 10^{6}$ to $1.8 \times 10^{7}$ years. The uncertainties of the $\log (E W)$ converted into log age uncertainties are listed in Table A.2. These values were determined using linear interpolation between the log $E W$ uncertainty limits and their corresponding log ages given by the evolution track. We found that an uncertainty of $\log E W$ of $0.1 \AA$ for $\log (E W)=1.9$ corresponds to an uncertainty in age of $0.16 \mathrm{Myr}$ for $6 \mathrm{Myr}$. The errors range from $2.14 \%$ to $6.59 \%$ with a mean value of $3.7 \%$, the highest value corresponding to $\log (t)=6.63$. The number of ionizing photons of the SB99 model is 3 orders of magnitude lower than that of the HII region with the same mass and ages varying between $4 \times 10^{6}$ and $2 \times 10^{7} \mathrm{yr}$. This decrease in the number of photons in the SB99 model generates uncertainties in the derived ages of the older regions. However, these uncertainties are not greater than $7 \%$.

Figure 2 we show the evolution of $(B-V)$ and $(V-I)$ colour model, computed with SB99, in the age range of $1 \times 10^{4}$ to $1 \times$ $10^{8}$ years and the theoretical colour-colour-ages for the cluster: $10^{4}, 10^{6}, 2.5 \times 10^{6}, 5 \times 10^{6}, 7.5 \times 10^{6}, 10^{7}, 5 \times 10^{7}, 10^{8}$ years. Note that increasing ages run anticlockwise. The $A_{V}=1 \mathrm{mag}$ reddening vector is located in the low right corner of the plot. We used the galactic reddening law $A_{V}=3.1 E(B-V)$ (Savage \& Mathis 1979).

The most of the colours of the observed HII regions fall far beyond the theoretical curve. This is most probably due to the internal reddening of the parent galaxy and that of the HII region. The internal reddening of an HII region can be determined 


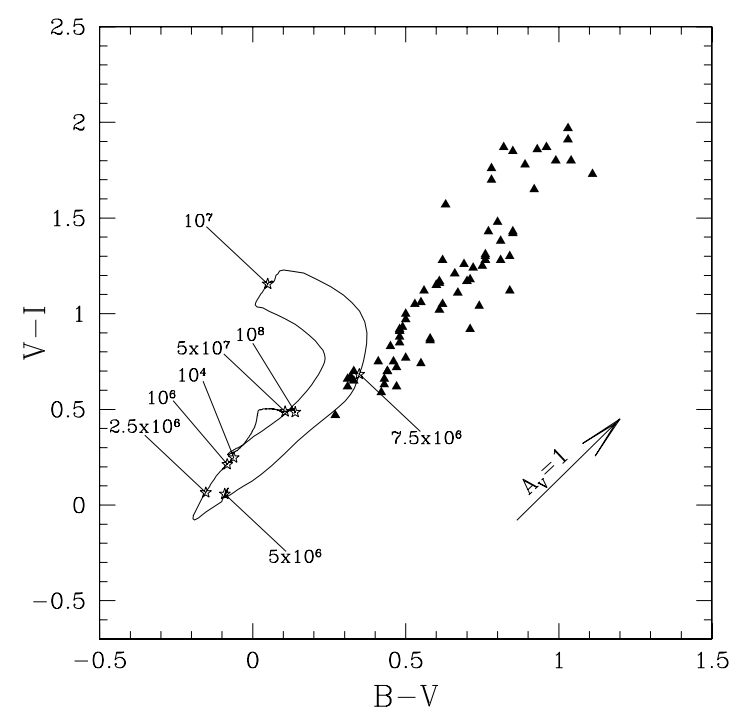

Fig. 2. Colour-colour diagram of the HII regions. The continuous line corresponds to the evolutionary track $(B-V)$ vs. $(V-I)$, determined by the Starburst 99 model. Open stars on the continuous line mark the ages $10^{4}, 10^{6}, 2.5 \times 10^{6}, 5 \times 10^{6}, 7.5 \times 10^{6}, 10^{7}, 5 \times 10^{7}, 10^{8}$ years for the clusters. The arrow (located below to the right) represents the value and the direction of the $A_{V}=1$ mag reddening. The filled triangles correspond to the colours of the observed HII regions.

comparing their colours with those of the SB99 models. Many of our observed regions have sizes comparable with the seeing, which can affect the colours given that the compared areas are not the same. Some regions were ruled out since they are close to the limit of resolution (seeing). Therefore, we selected regions with a limiting size $30 \%$ larger than the seeing to estimate the internal HII regions reddening. We were able to determine the total internal reddening for 73 regions. It was estimated as the distance of the observed colours to the points on the theoretical curve, which was determined by moving the observed point parallel to the reddening vector, until it crossed the evolutionary track. The estimated $E(B-V)$ value for all regions are listed in Table A.1. In addition we inferred the age for these regions from the colour-colour evolutionary track and compared it with those determined using the $E W(\mathrm{H} \alpha)$. In Fig. 7 we show the percentage difference between $\operatorname{Age}(E W(\mathrm{H} \alpha))$ and Age(colour-colour) relative to $\operatorname{Age}(E W(\mathrm{H} \alpha))$ sorted by increasing right ascension. The new estimates are in good agreement with most of the observed HII regions. We find that the differences between ages range between $-16 \%$ to $35 \%$ with an average value of $13 \%$.

The $\mathrm{H} \alpha$ luminosity was corrected for internal reddening using the estimated $E(B-V)$ value for each region (see Table A.1). Moreover, the $E W$ of $\mathrm{H} \alpha$ and HII region colours are given by SB99 as a function of time, which in turn give the evolutionary track of the luminosity of the emission lines. This luminosity strongly depends on the age and mass of the cluster. In Figs. A.1b to A.7b, we show the reddening free $\mathrm{H} \alpha$ luminosity as a function of age for different HII region masses. The continuous line from bottom to top corresponds to the evolutionary track of the H $\alpha$ luminosity for an HII region with $10^{5}, 10^{6}, 10^{7}$, $10^{8}$, and $10^{9}$ solar masses. Evolutionary track of the $\mathrm{H} \alpha$ luminosity for HII region with masses $3 \times 10^{5}, 3 \times 10^{6}, 3 \times 10^{7}$, and $3 \times 10^{8} M_{\odot}$ (dashed line) and masses $5 \times 10^{5}, 5 \times 10^{6}, 5 \times 10^{7}$, and $5 \times 10^{8} M_{\odot}$ (short dashed lines) were plotted. whenever possible, the nucleus of the galaxies of the sample (see Sect. A) was indicated in the graphics. If we assume that the SB99 model can reproduce the observed HII region properties such as luminosity, $E W$, colours, etc, we can infer that this model can also give us an estimation of the stellar mass of the ionizing cluster of the sample. Therefore, from the position of the objects in Figs. A.1b to A.7b we conclude that clusters with masses higher than $10^{6} M_{\odot}$ are associated with the observed HII region. The adopted cluster mass was assumed to be that of the luminosity track closest to the HII region. For a few cases with a low $\mathrm{H} \alpha$ emission and without dereddening, we determined the mass from the track of the blue absolute luminosity also calculated with the SB99 model. It seems that the mass range for the HII regions is $5 \times 10^{5}<M / M_{\odot}<1 \times 10^{9}$. Most regions have masses between $1 \times 10^{6}<M / M_{\odot}<3 \times 10^{7}$. We observed 9 HII regions with masses higher than $1 \times 10^{8} M_{\odot}$. The mass of each HII region is listed in Table A.2.

\section{Nature of the star-forming regions}

In this section, we discuss the nature of the star-forming regions detected in the sample of minor mergers. The parameters used in this analysis are i) blue absolute magnitude $M_{B}$, ii) $\mathrm{H} \alpha$ luminosity $\mathcal{L}(\mathrm{H} \alpha)$, iii) star formation rate $S F R(\mathrm{H} \alpha)$, and iv) mass of the stellar cluster $M$.

The integrated blue absolute magnitude $M_{B}$ of the HII region listed in Table A. 1 corresponds to the whole region and is corrected by the contribution of the underlying disk. For more details, see FP2004. The distribution of $M_{B}$ is shown in Fig. 6. The magnitude limit of the TDG observed at or near the tip of long tidal tails (Hunter et al. 2000; Weilbacher et al. 2004). Note that $62 \%$ of the HII regions have $M_{B} \leq-15$, and 2 of them have magnitude $M_{B} \leq-19$. It is important to point out that these HII regions are more luminous (in $M_{B}$ ) than the average starforming regions in normal galaxies and than the TDG candidates of the sample of Weilbacher et al. (2000), whose magnitude limit is $M_{B}=-17.1$. In addition, Weilbacher et al. (2000) find that the TDG candidates are, on average, 4 mag more luminous than the HII regions reported by Bresolin \& Kennicutt (1997). Some observed HII regions are brighter than $M_{B}>-19$ and are as bright as the TDG on the edge of the tidal tail of Arp 105 (Mirabel et al. 1992; and Duc \& Mirabel 1994).

The H $\alpha$ luminosity of the HII regions in our sample is, in general, much brighter than the brightest regions observed in spiral galaxies. In Fig. 3, we plotted the $\mathrm{H} \alpha$ luminosity dereddening distribution of our HII region sample. The histogram was plotted taking bins of $\log (\mathcal{L}(\mathrm{H} \alpha))=0.2\left(\left[\operatorname{erg~s}^{-1}\right]\right)$. We then compared it with the TDGs sample of Temporin et al. ${ }^{3}$ (2003) in Fig. 3. Forty-three HII regions of our sample have $\mathrm{H} \alpha$ luminosity brighter than $10^{40} \mathrm{erg} \mathrm{s}^{-1}$.

Figure 4 shows the mass distribution of the HII regions estimated from the SB99 model (see Sect. 3) obtained by the $\mathrm{H} \alpha$ luminosity (upper panel, right shade) and obtained by the blue absolute magnitude (lower panel, left shade). The histogram bins are $M=0.2 M_{\odot}$. We exclude the nucleus of the galaxy sample (see Sect. A) in the distribution of mass. We can see a large number of regions (32) with $1 \times 10^{6}<M / M_{\odot}<3 \times 10^{7}$ and 9 of them with masses higher than $1 \times 10^{8}<M / M_{\odot}$.

\section{Conclusions}

We have presented an analysis of the nature of 116 starforming regions, observed in a sample of 11 pairs of minor mergers galaxies. We found that $62 \%$ of the HII regions have

\footnotetext{
${ }^{3}$ Detailed study of the group ultra-compact CG J1720-67.8, which contains candidates to TDGs.
} 


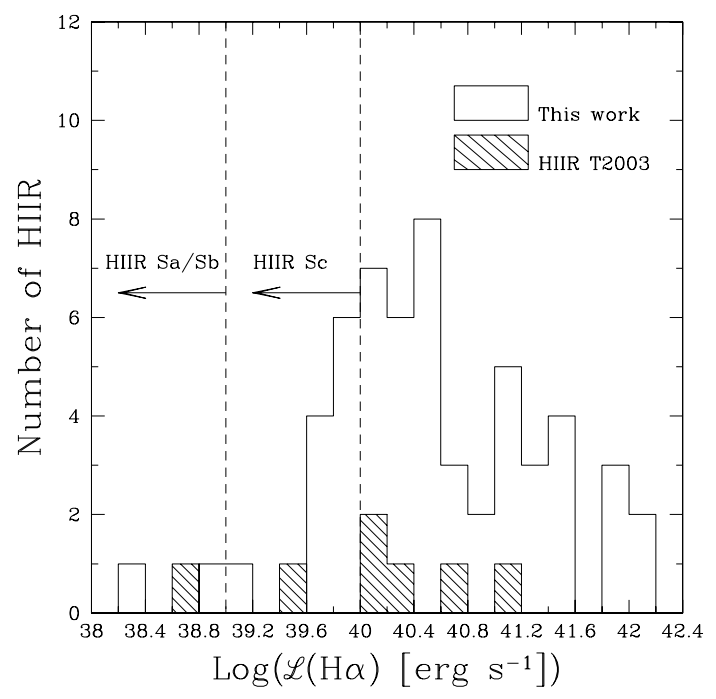

Fig. 3. Distribution of $\mathrm{H} \alpha$ luminosity-free reddening of the star-forming regions of our sample (white area) and the sample of Temporin et al. (2003) (shadowed area). The dotted lines indicate the limits in $\mathrm{H} \alpha$ luminosity of HII regions observed in normal galaxies with morphological type $\mathrm{Sa} / \mathrm{Sb}$ and $\mathrm{Sc}$.
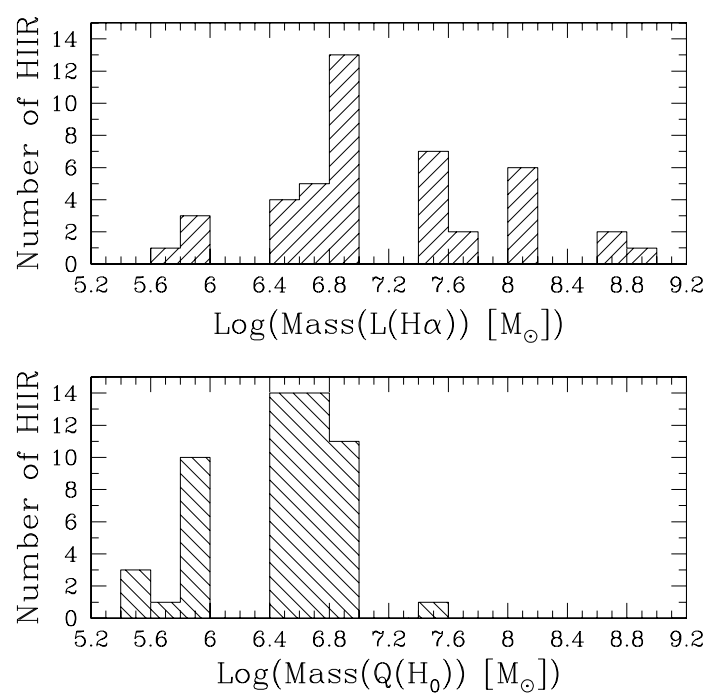

Fig. 4. Distribution of mass for the HII region of the sample obtained by the $\mathrm{H} \alpha$ luminosity (upper panel) and obtained by the blue absolute magnitude (lower panel). We exclude the nucleus and suspected nucleus of the sample. The histogram was constructed taking bins of $M=0.2 M_{\odot}$.

$M_{B} \leq-15$ mag, which is the upper limit of GHIIC observed by Bresolin \& Kennicutt (1997) in Sa/Sb type galaxies. We pointed out that 5 regions listed in Table A.1 are six times brighter than the TDG candidates of the Weilbacher et al. (2000) sample and are as bright as those observed in the tidal tail of the Arp 105 system.

We also found that 43 regions have $\mathrm{H} \alpha$ luminosities brighter than $10^{40} \mathrm{erg} \mathrm{s}^{-1}$, which is the upper luminosity limit for HII regions in normal Sc galaxies. We found that $48 \mathrm{HII}$ regions have $S F R>0.1 M_{\odot} \mathrm{yr}^{-1}$ (typical of TDGs). Very high SFR are found for 9 HII regions: $S F R>1 M_{\odot} \mathrm{yr}^{-1}$, an SRF that is higher than the average value for the sample of Temporin et al. (2003).

We have estimated the ages and the masses for HII regions of the sample using the synthesis models of Leitherer et al. (1999). The average age of the HII region is $t=6.03 \times 10^{6}$ years. We observed that $32 \mathrm{HII}$ regions have an estimated mass in the range
$1 \times 10^{6}<M / M_{\odot}<3 \times 10^{7}$. These regions have masses comparable to YMC. In our sample we have detected 9 regions associated with very massive stellar clusters, with masses greater than $1 \times 10^{8} M_{\odot}$. These regions are more massive than the YMCs found in the outer regions of the galactic disk or in the tidal tails of the NGC 6872. This result suggests that these HII regions are GHIICs or they could be pre-TDG candidates. High-resolution images and the spectra of the objects studied here are required for confirm whether those regions are real TDGs or very luminous HII region complexes.

Acknowledgements. This work has been partially supported by CONICET and CNPq (PRONEX 66.2088/1997-2).

\section{References}

Arp, H., \& Madore, B. F. 1987, A Catalogue of Southern Peculiar Galaxies and Associations (Cambridge: Cambridge Univ. Press).

Beckman, J. E., Rozas, M., \& Knapen, J. H. 1998, PASA, 15, 83

Beckman, J. E., Zurita, A., Rozas, M., Cardwell, A., \& Relaño 2002, RMxAC, 12,213

Braine, J., Duc, P.-A., Lisenfeld, U., et al. 2001, A\&A, 378, 51

Bresolin, F., \& Kennicutt, R. C. 1997, AJ, 113, 975

Cortese, L., Gavazzi, G., Boselli, A., \& Iglesias-Paramo, J. 2004, A\&A, 416, 119 de Grijs, R., Lee, J. T., Clemencia Mora Herrera, M., Fritze-v. Alvensleben, U., \& Anders, P. 2003, New Astron., 8, 155

Duc, P.-A., Bournaud, F., \& Masset, F. 2004, A\&A, 427, 803

Duc, P.-A., \& Mirabel, I. F. 1994, A\&A, 289, 83

English, J., Norris, R. P., Freeman, K. C., \& Boot, R. S. 2003, AJ, 125, 1134

Ferreiro, D. L., \& Pastoriza, M. G. 2004, A\&A, 428, 837 (FP2004)

Gallagher, S. C., Charlton, J. C., Hunsberger, S. D., Zaritsky, D., \& Whitmore, B. C. 2001, AJ, 122,163

Gerhard, O., Arnaboldi, M., Freeman, K. C., \& Okamura, S. 2002, ApJ, 580, L121

Gilbert, A. M., Graham, J. R., McLean, I. S., et al. 2000, ApJ, 533, L57

Giammanco, C., Beckman, J. E., \& Cedreś, B. 2005, A\&A, 438, 599

Girardi, L., Bica, E., Pastoriza, M. G., \& Winge, C. 1997, ApJ, 486, 847

Hameed, S., \& Devereux, N. 2005, AJ, 129, 259

Hibbard, J., \& Barnes, J. E. 2004, Recycling intergalactic and interstellar matter, IAU Symp., 217, 510

Holtzman, J. A., Faber, S. M., Shaya, E. J., et al. 1992, AJ, 103, 691

Hoopes, C., \& Walterbos, R. M. 2003, ApJ, 586, 902

Hunter, D. A., Hunsberger, S. D., \& Roye, E. W. 2000, ApJ, 542, 137

Iglesias-Páramo, J., \& Vílchez, J. M. 2001, ApJ, 550, 204

Kennicutt, R. C. Jr., Edgar, B. K., \& Hodge, P. W. 1989, ApJ, 337, 761

Kennicutt, R. C. Jr., \& Kent, S. M. 1983, AJ, 88, 1094

Kennicutt, R. C., Tamblyn, P., \& Congdon, C. W. 1994, ApJ, 435, 22

Kennicutt, R. C. 1998, ARA\&A, 36, 189

Knierman, K. A., Gallagher, S. C., Charlton, J. C., et al. 2003, AJ, 126, 1227

Leitherer, C., Schaerer, D., Goldader, J. D., et al. 1999, ApJS, 123, 3

López-Sánchez, A. R., Esteban, C., \& Rodríguez, M. 2004, ApJS, 153, 243

Madau, P., Pozzetti, L., \& Dickinson, M. 1998, ApJ, 498, 106

Mendes de Oliveira, C., Cypriano, E. S., Sodre, L. Jr., \& Balkowski, C. 2004, ApJ, 605, L17

Mihos, C. 2004, Recycling intergalactic and interstellar matter, IAU Symp., 217, 390

Mirabel, I. F., Dottori, H., \& Lutz, D. 1992, A\&A, 256, L19

Osterbrock, D. E. 1989, Physics Today, 42, 123

Pastoriza, M. G., Donzelli, C. J., \& Bonatto, C. 1999, A\&A, 347, 55

Ryan-Weber, E. V., Meurer, G. R., Freeman, K. C., et al. 2004, AJ, 127, 1431

Savage, B. D., \& Mathis, J. S. 1979, ARA\&A, 17, 73

Saviane, I., Hibbard, J. E., \& Rich, R. M. 2004, AJ, 127, 660

Schweizer, F., Miller, B. W., Whitmore, B. C., \& Fall, S. M. 1996, AJ, 112, 1839

Temporin, S., Weinberger, R., Galaz, G., \& Kerber, F. 2003, ApJ, 587, 660

Tran, H. D., Sirianni, M., Ford, H. C., et al. 2003, ApJ, 585, 750

Weilbacher, P. M., Duc, P.-A., Fritze v. Alvensleben, U., Martin, P., \& Fricke, K. J. 2000, A\&A, 358, 819

Weilbacher, P. M., Duc, P.-A., \& Fritze-v. Alvensleben, U. 2003, Ap\&SS, 284, 639

Weilbacher, P. M., Fritze-v. Alvensleben, U., \& Duc, P.-A. 2004, IAUS, 217, 540 Whitmore, B. C., Zhang, Q., Leitherer, C., et al. 1999, AJ, 118, 1551

Zepf, S. E., Ashman, K. M., English, J., Freeman, K. C., \& Sharples, R. M. 1999, AJ, 118, 752

Zurita, A., Rozas, M., \& Beckman, J. E. 2000, A\&A, 363, 9 
D. L. Ferreiro et al.: Modelling HII region properties $(R N)$, Online Material $p 1$

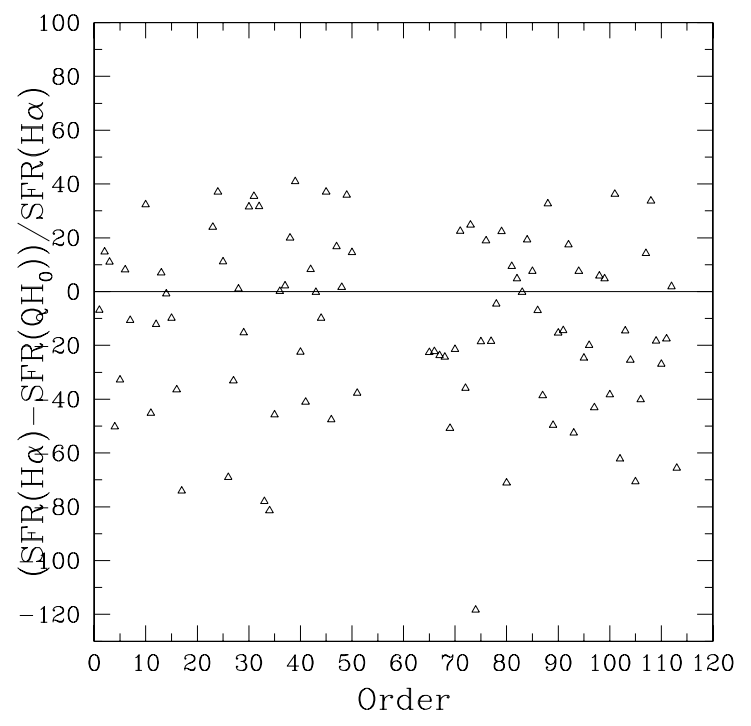

Fig. 5. Percentage difference between $\operatorname{SFR}(\mathrm{H} \alpha)$ and $\operatorname{SFR}\left(Q \mathrm{H}_{0}\right)$ relative to $\operatorname{SFR}(\mathrm{H} \alpha)$ for the regions with $\mathrm{H} \alpha$ calibration ordered according to increasing right ascension.

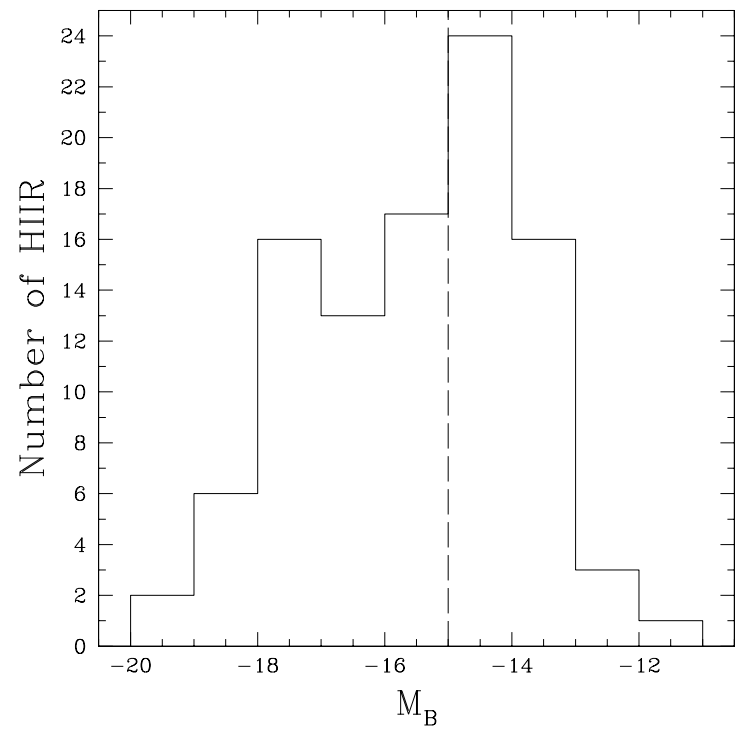

Fig. 6. Distribution of luminosity of the star-forming regions of the sample. The dashed vertical line indicates the magnitude limit of the TDG observed at or near the tip of long tidal tails.

\section{Appendix A: The galaxies}

In this section, we describe the main properties of the HII regions in the individual galaxies. Figures A.1 to A.7 show the evolutionary tracks of $\mathrm{H} \alpha$ luminosity with age $(\mathcal{L}(\mathrm{H} \alpha)$ vs. $t)$ for different masses as described in Sect. 3.2.

AM1256-433: In the main component of this interacting pair $7 \mathrm{HII}$ regions in the $\mathrm{H} \alpha$ image were observed (see Fig. A.1a). The ages are between $5.6 \times 10^{6}$ to $6.0 \times 10^{6}$ years. Figure A. 1 b shows $\mathcal{L}(\mathrm{H} \alpha)$ vs. $t$ for HII regions of the main component (solid triangle). We can see that the HII regions have masses between $5 \times 10^{6}<M / M_{\odot}<3 \times 10^{7}$. Two regions, \#5 and \#6, have masses $M=3 \times 10^{7} M_{\odot}$, and their colours are similar $\left((B-V)_{0} \sim-0.06\right)$. Given its relative position in the galaxy and its colours, region \#5 (open circle and labeled $N_{A}$ ?) may correspond to the nucleus of the main galaxy.

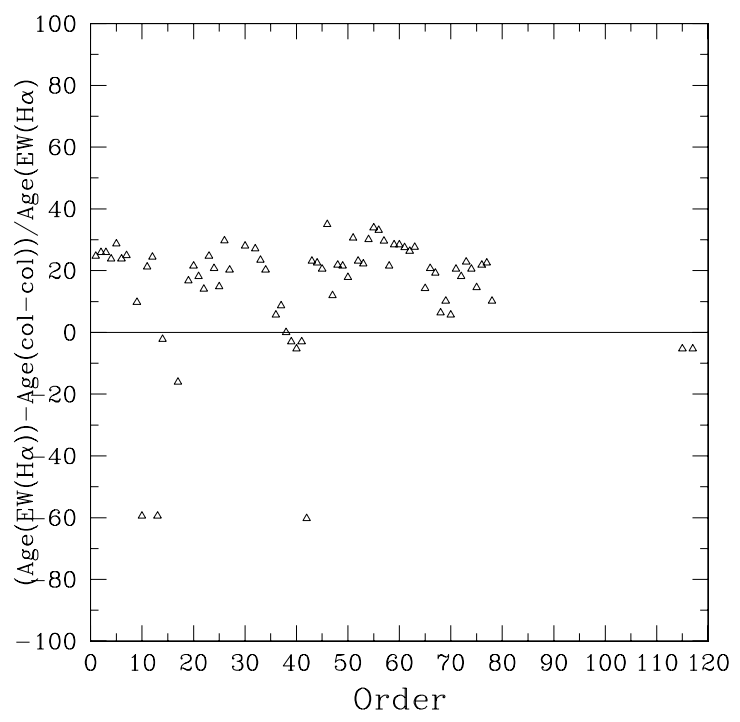

Fig. 7. Percentage difference between $\operatorname{Age}(E W(\mathrm{H} \alpha))$ and Age(colour-colour) relative to $\operatorname{Age}(E W(\mathrm{H} \alpha))$ for the regions internal reddening estimated ordered according to increasing right ascension.

We were not able to estimate the mass of the HII region in the secondary component because we do not have $\mathrm{H} \alpha$ calibration for this component. However, a rough estimate of its mass could be made using the $M_{B}$ vs. $t$ relation. An approximate mass for the central region is $\sim M=5 \times 10^{6} M_{\odot}$.

AM1401-324: The main galaxy of this pair has a very bright nucleus and several strong HII regions (see Fig. A.2a). The masses are in the range $3 \times 10^{6}<M / M_{\odot}<1 \times 10^{9}$ (solid triangles in Fig. A.2b). The primary component has three very massive HII regions. Two of them, $N_{A}$ and \#3, with masses $M=1 \times 10^{9} M_{\odot}$, colours $(B-V) \sim 0.05$, and age of $1 \times 10^{7}$ years. The region \#2 has a mass $M=1 \times 10^{8} M_{\odot}$ and is the youngest of the three regions ( $t=5.7 \times 10^{6}$ years). HII regions \#2 and \#3 are located in each extreme of the galaxy bar(see Fig. A.2a).

The companion is located at the end of the main galaxy arm and seems to be a dwarf elliptical. The nucleus mass was estimated to be $M=3 \times 10^{7} M_{\odot}$. In Fig. A. $2 \mathrm{~b}$ we can see a clear difference in age between the nuclei, about $\Delta t=3 \mathrm{Myr}$.

AM1448-262: We do not have $\mathrm{H} \alpha$ calibration for the two components. However, we can estimate the mass of the HII region using the relation $M_{B}$ vs. $t$ for these regions. The masses of the HII regions in the main component are in the range $3 \times 10^{5}$ and $3 \times 10^{6} M_{\odot}$. We do not have enough information to estimate the location of the nuclear region in this component. In the secondary component, the nuclear region has an approximate mass $\sim M=1 \times 10^{7} M_{\odot}$.

AM2030-303: This system is probably composed of two or more galaxies. Two HII regions were observed in the main component of the pair (see Fig. A.3a). Region \#1, at the north of the nucleus, is the largest and the most $\mathrm{H} \alpha$ luminous; its mass is $M=1 \times 10^{8} M_{\odot}$. Region \#2 has a mass $M=1 \times 10^{7} M_{\odot}$ (see Fig. A.3b solid triangle points).

We could detect four star-forming regions in the secondary component (see Fig. A.3b). The most massive is \#3, with a mass of $M=3 \times 10^{7} M_{\odot}$ and age of $t=6.31 \times 10^{6}$ years. The other three HII regions (open stars) have masses between $5 \times 10^{5}<$ $M / M_{\odot}<3 \times 10^{6}$ but they have very different ages $\left(4.21 \times 10^{6}<\right.$ $t<6.31 \times 10^{6}$ years) (see Fig. A.3b).

AM2058-381: This pair consists of a main galaxy with two spiral arms and a companion in the extreme of the southern arm. 


\section{L. Ferreiro et al.: Modelling HII region properties $(R N)$, Online Material $p 2$}

We can detect a nucleus and eleven HII regions in the main component (see Fig. A.4a). The nucleus has a mass $M=5 \times 10^{8} M_{\odot}$ and age of $t=1 \times 10^{7}$ years (see Fig. A.4b, open circle labelled $N_{A}$ ). Regions \#1 to \#4 and \#6 have masses between $\times 10^{6}<$ $M / M_{\odot}<3 \times 10^{6}$; regions \#5 and \#7 to \#11 have a mass range between $5 \times 10^{6}<M / M_{\odot}<3 \times 10^{7}$ (solid triangles). The ages of the regions are between $5.2 \times 10^{6}<t<6.7 \times 10^{6}$ years (see Fig. A.4b).

The nuclear region of the secondary component is a little more massive $\left(M=1 \times 10^{8} M_{\odot}\right)$ than the HII regions of the other component, but the age $t=3 \times 10^{7}$ years is similar (see Fig. A.4b open circle labeled $N_{B}$ ).

AM2105-332: In this pair we could not detect $\mathrm{H} \alpha$ emission out of the nuclei (see Fig. A.5a). The nucleus of the main companion is fifty percent more massive but much older than the other nucleus (see Fig. A.5b open circle). Its mass is $M=$ $5 \times 10^{6} M_{\odot}$ and the age is $t=1.3 \times 10^{7}$ years. The nucleus of the secondary has a mass $M=1 \times 10^{6} M_{\odot}$ and $t=6.5 \times 10^{6}$ years old.

AM2229-735: Using the $\mathrm{H} \alpha$ image (see Fig. A.6a), we detected in the primary component a nucleus and six HII regions. The nuclear region is very massive, its mass is $M=5 \times 10^{8} M_{\odot}$ (see Fig. A.6b open circle labelled $N_{A}$ ). The HII regions have masses $M \sim 1 \times 10^{8} M_{\odot}$, but, region \#3 is the least massive; with a mass $M=1 \times 10^{7} M_{\odot}$ (solid triangle).

In the secondary component we can detect $\mathrm{H} \alpha$ emission only in the nuclear region (see Fig. A.6a). Its mass is $M=3 \times 10^{8} M_{\odot}$ (open star labeled $N_{B}$ ) and it could be connected to the main galaxy by a bridge. On the tip of the bridge we can observe region \#2 (see Fig. A.6a), the youngest of the pair $(t=5.1 \times$ $10^{6}$ years), with a mass $M=5 \times 10^{7} M_{\odot}$. All the regions, including the nucleus, have ages between $5 \times 10^{6}<t<6.4 \times 10^{6}$ years (see Fig. A.6b).

AM2238-575: This interacting pair is formed by a giant $\mathrm{Sb} / \mathrm{c}$ peculiar galaxy and the secondary is an edge-on disk galaxy, probably SO type. The main galaxy does not have $\mathrm{H} \alpha$ emission in the nuclear region. However, we could detect twelve HII regions around it. A rough estimate of its mass was determined using the relation $M_{B}$ vs. $t$ for each region. The HII regions may have a mass between $5 \times 10^{5}$ to $1 \times 10^{7} M_{\odot}$.

We could not detect $\mathrm{H} \alpha$ emission out of the nuclear region. This region has an estimated mass $M=5 \times 10^{7} M_{\odot}$.
AM2306-721: This pair is formed by a peculiar spiral with disturbed arms interacting with an irregular galaxy, probably disrupted by tidal forces (see Fig. A.7a). In the $\mathrm{H} \alpha$ image we detected a nucleus and ten HII regions. The nucleus has a mass $M=1 \times 10^{8} M_{\odot}$ and its age is $t=6.7 \times 10^{6}$ years (see Fig. A. $7 \mathrm{~b}$ open circle labelled $N_{A}$ ). The HII regions in the main component (solid triangles) have masses between $5 \times 10^{6}<M / M_{\odot}<5 \times 10^{7}$. The most massive is the region \#6 $\left(M=5 \times 10^{7} M_{\odot}\right)$, and regions $\# 1$, \#2, and \#7 to \#10 have masses between $1 \times 10^{7}<M / M_{\odot}<$ $3 \times 10^{7}$. The ages of all regions are between $5.7 \times 10^{6}<t<$ $6.5 \times 10^{6}$ years.

The main body of the secondary galaxy is resolved into three bright HII regions (see Fig. A.7a). Their masses are approximately $M \sim 5 \times 10^{8} M_{\odot}, 10$ times more massive than the observed regions in the main component. The ages are $t \sim$ $6 \times 10^{6}$ years (see Fig. A.7b open star). Region \#2 (open circle labeled $N_{B}$ ) is a little older than regions \#1 and \#3, and it may be the nuclear region in this component.

AM2322-821: The main component is a $\mathrm{Sa} / \mathrm{b}$ galaxy with an irregular companion. We detected a large number $(n=27)$ of HII regions in the $\mathrm{H} \alpha$ image. A rough estimate of its mass was determined using the relation $M_{B}$ vs. $t$ for each region. The nuclear region is at least 10 times more massive than the HII regions observed in the galaxy. This region has $M=1 \times 10^{9} M_{\odot}$ and its age is $t=6.7 \times 10^{6}$ years. In this galaxy the HII regions have a total mass estimated between $1 \times 10^{6}<M / M_{\odot}<3 \times 10^{7}$. The most massive HII region is \#1 and the least massive is \#25. The ages of the regions is between $5.2 \times 10^{6}<t<6.7 \times 10^{6}$ years.

Many HII regions were observed in the secondary galaxy. The total number is seven, six are located, in the diagram $\mathcal{L}(\mathrm{H} \alpha)$ vs. $t$, in the same zone as the regions in the main component. Region \#1 is more massive and older than the other six regions. Its mass and age are $M=3 \times 10^{7} M_{\odot}$ and $t=7.38 \times 10^{6}$ years, respectively. Having analysed the colour $\left((B-V)_{0}=0.33\right)$, mass, and age, we suspect that this region could be the nuclear region in this galaxy.

AM2330-451: This pair is formed by an SO galaxy and its companion is a disk galaxy. In the main component we could not detect $\mathrm{H} \alpha$ emission out of the nuclear region, which has an estimated mass $M=5 \times 10^{7} M_{\odot}$. We detect two HII regions in the secondary component, and the masses are $M \sim 1 \times 10^{6}$ and $M \sim 3 \times 10^{5} M_{\odot}$. 


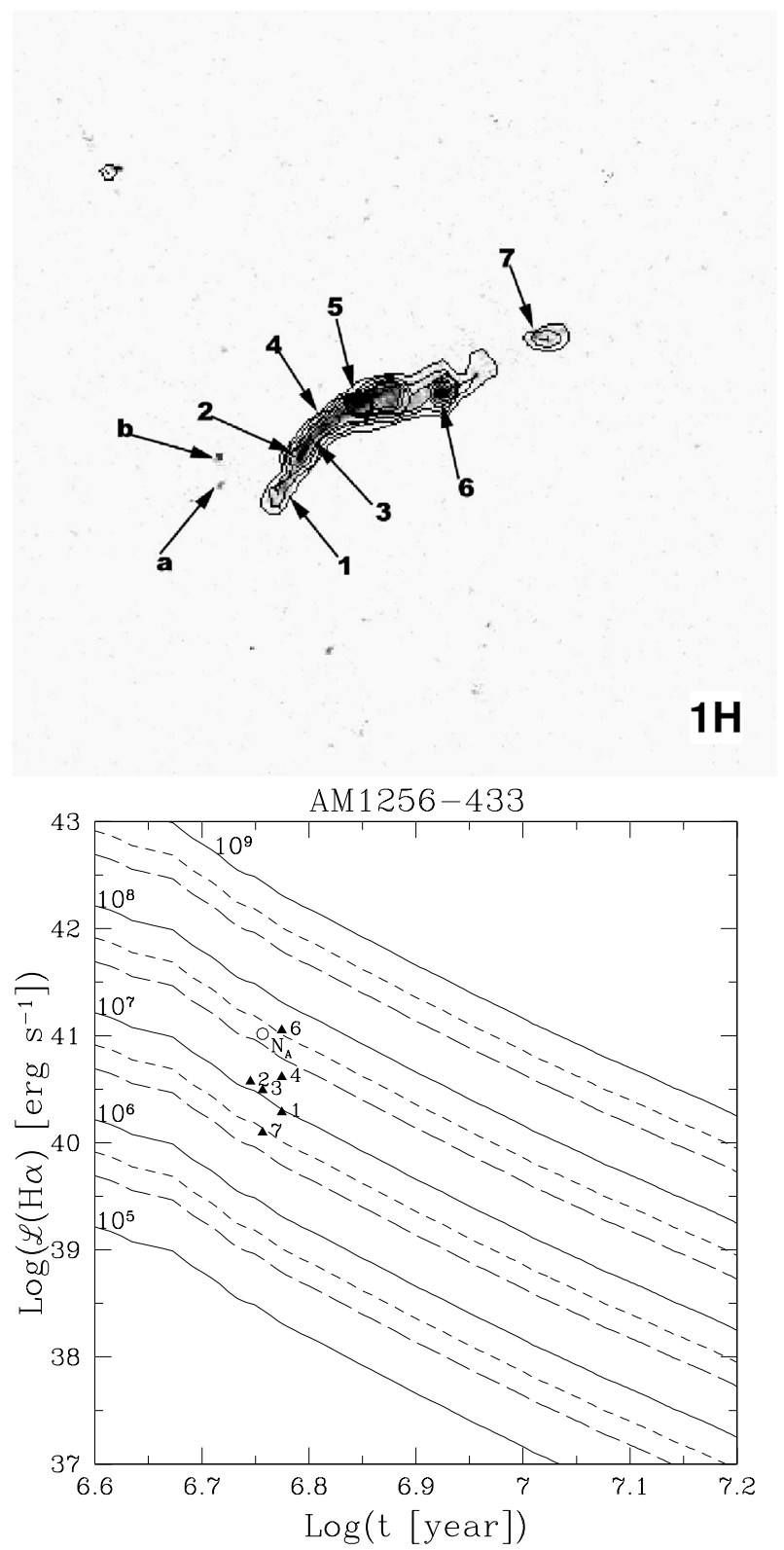

Fig. A.1. AM1256-433. Top panel a): $\mathrm{H} \alpha$ image and contours map. Bottom panel b): $\mathcal{L}(\mathrm{H} \alpha)$ evolutionary track for $\mathrm{HII}$ regions of the pair with masses between $1 \times 10^{5}<M / M_{\odot}<1 \times 10^{9}$ determined by Starburst 99 model. The continuous lines from bottom to top correspond to the evolutionary track of the $\mathrm{H} \alpha$ luminosity for HII regions with $10^{5}$, $10^{6}, 10^{7}, 10^{8}$, and $10^{9}$ solar masses. The dashed lines from the bottom correspond to HII regions with $3 \times 10^{5}, 3 \times 10^{6}, 3 \times 10^{7}$, and $3 \times 10^{8}$ solar masses, and the short dashed lines correspond to HII region with $5 \times 10^{5}, 5 \times 10^{6}, 5 \times 10^{7}$, and $5 \times 10^{8}$ solar masses. The filled triangles correspond to $\mathrm{H} \alpha$ luminosity (corrected by internal reddening) vs. age of the HII regions and the open stars correspond to the nucleus of the host galaxy.

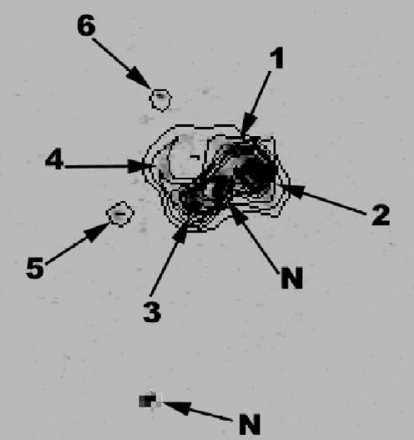

$2 \mathrm{H}$

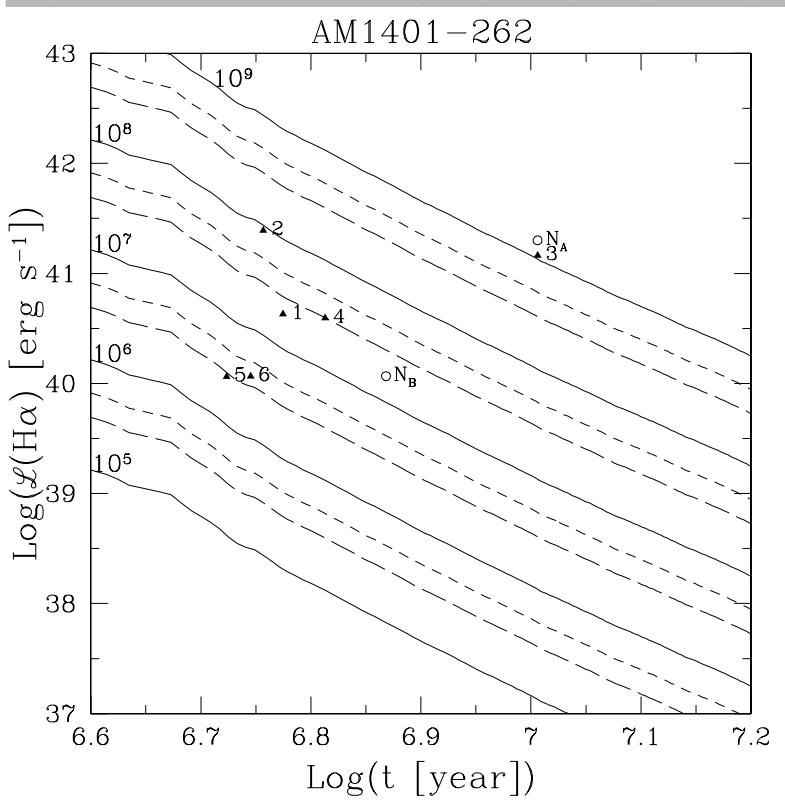

Fig. A.2. AM1401-324. Same as Fig. A.1. 
D. L. Ferreiro et al.: Modelling HII region properties $(R N)$, Online Material p 4

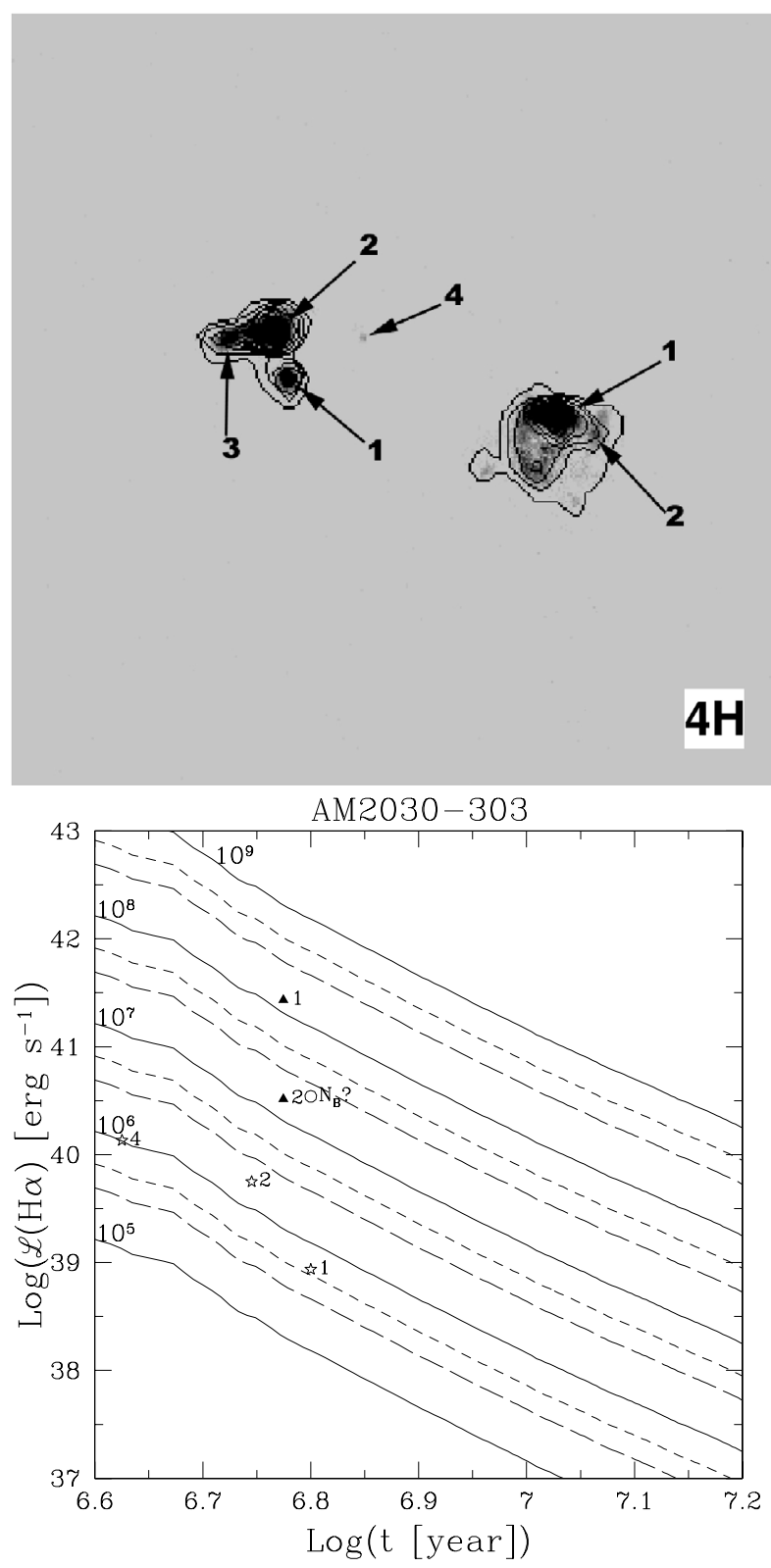

Fig. A.3. AM2030-303. Same as Fig. A.1.
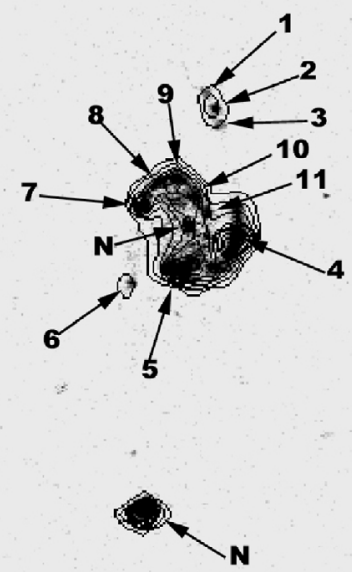

$5 \mathrm{H}$

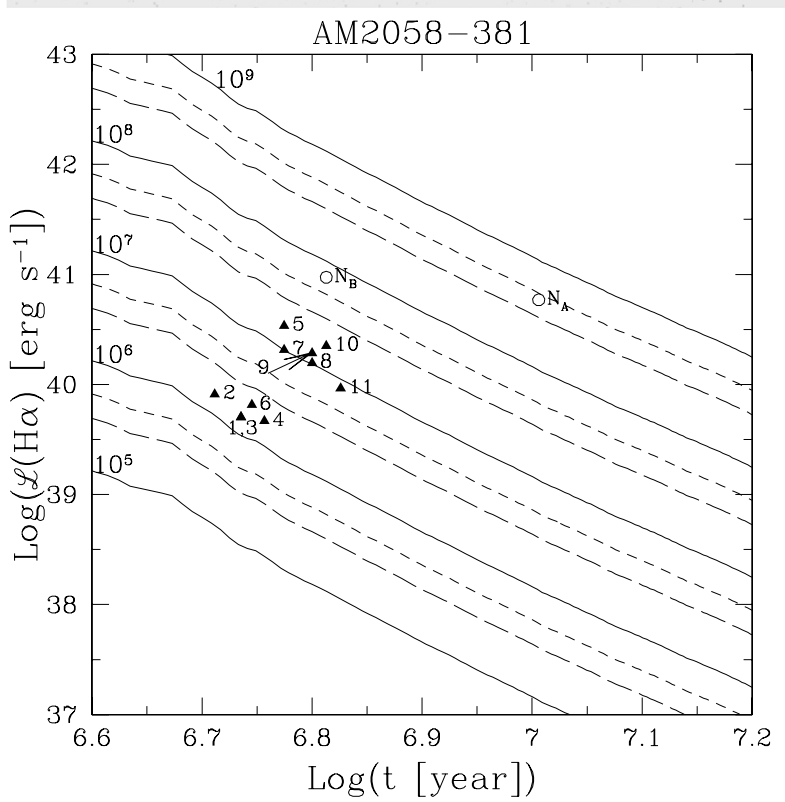

Fig. A.4. AM2058-381. Same as Fig. A.1. 
D. L. Ferreiro et al.: Modelling HII region properties $(R N)$, Online Material p 5

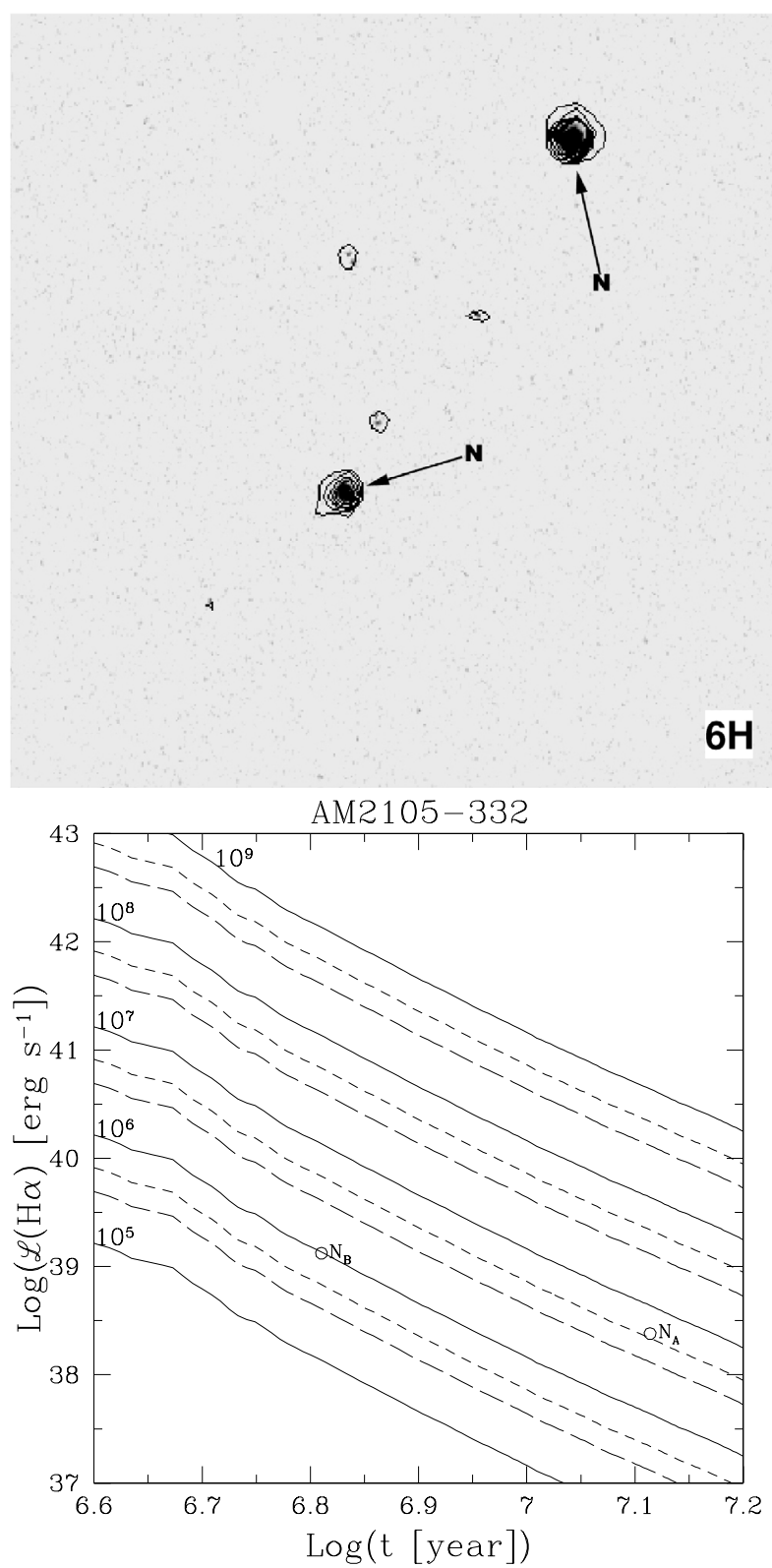

Fig. A.5. AM2105-332. Same as Fig. A.1.

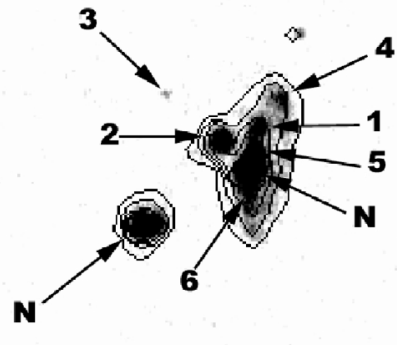

7H

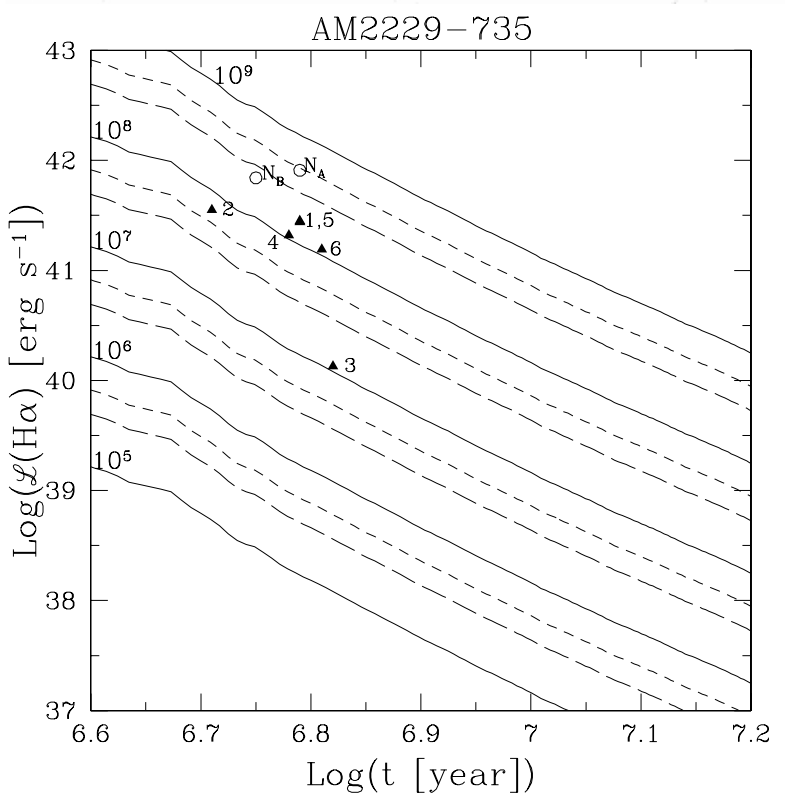

Fig. A.6. AM2229-735. Same as Fig. A.1 
D. L. Ferreiro et al.: Modelling HII region properties $(R N)$, Online Material p 6
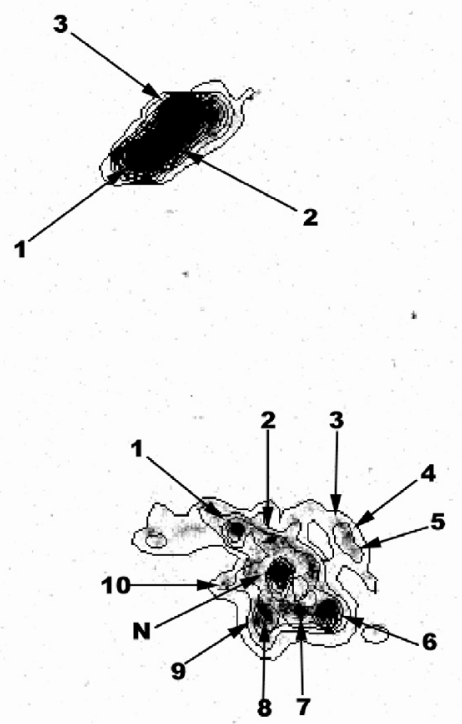

$9 \mathrm{H}$

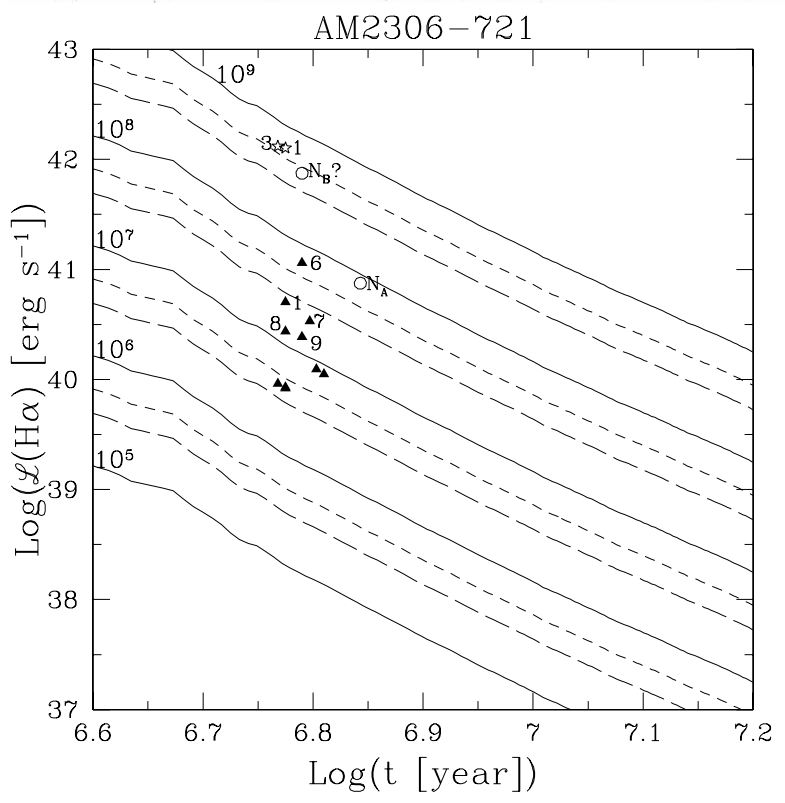

Fig. A.7. AM2306-721. Same as Fig. A.1. 
D. L. Ferreiro et al.: Modelling HII region properties $(R N)$, Online Material p 7

Table A.1. HII region properties in a sample of minor merger I: Region, Arp galaxy, right ascension and declination, blue absolute magnitude, integrated colours, and internal reddening.

\begin{tabular}{|c|c|c|c|c|c|c|c|}
\hline $\begin{array}{l}\text { Reg. } \\
\text { No. }\end{array}$ & $\begin{array}{c}\text { Galaxy } \\
\text { Arp }\end{array}$ & $\begin{array}{c}\alpha \\
\mathrm{J} 2000\end{array}$ & $\begin{array}{c}\delta \\
\mathrm{J} 2000\end{array}$ & $\begin{array}{c}M_{B} \\
{[\mathrm{mag}]}\end{array}$ & $\overline{\overline{B-V}}$ & $\overline{\overline{V-I}}$ & $\overline{E(B-V)}$ \\
\hline 7 & AM1256-433W & $12: 58: 54.5$ & $-43: 50: 17.0$ & $-15.69 \pm 0.27$ & 0.62 & 1.05 & 0.356 \\
\hline 6 & AM1256-433W & $12: 58: 56.2$ & $-43: 50: 26.9$ & $-17.54 \pm 0.27$ & 0.81 & 1.38 & 0.367 \\
\hline $5(\mathrm{~N} ?)$ & AM1256-433W & $12: 58: 57.7$ & $-43: 50: 29.3$ & $-17.36 \pm 0.27$ & 0.77 & 1.43 & 0.373 \\
\hline 4 & AM1256-433W & $12: 58: 58.3$ & $-43: 50: 34.0$ & $-16.74 \pm 0.27$ & 0.76 & 1.31 & 0.367 \\
\hline 3 & AM1256-433W & $12: 58: 58.7$ & $-43: 50: 37.6$ & $-16.10 \pm 0.27$ & 0.76 & 1.28 & 0.362 \\
\hline 2 & AM1256-433W & $12: 58: 58.8$ & $-43: 50: 40.4$ & $-15.83 \pm 0.27$ & 0.81 & 1.28 & 0.348 \\
\hline 1 & AM1256-433W & $12: 58: 59.0$ & $-43: 50: 45.9$ & $-16.12 \pm 0.27$ & 0.69 & 1.26 & 0.372 \\
\hline 2 & AM1256-433E & $12: 58: 59.6$ & $-43: 50: 34.6$ & $-16.70 \pm 0.27$ & 1.11 & 1.73 & 0.362 \\
\hline 1 & AM1256-433E & $12: 58: 59.8$ & $-43: 50: 47.0$ & $-19.80 \pm 0.27$ & 1.61 & 1.96 & 1.115 \\
\hline 2 & AM1401-324N & 14:04:16.9 & $-33: 01: 31.6$ & $-18.36 \pm 0.30$ & 0.33 & 0.70 & 0.352 \\
\hline 1 & AM1401-324N & $14: 04: 17.2$ & $-33: 01: 27.9$ & $-16.73 \pm 0.30$ & 0.31 & 0.66 & 0.352 \\
\hline $\mathrm{N}$ & AM1401-324N & $14: 04: 17.3$ & $-33: 01: 34.0$ & $-19.80 \pm 0.30$ & 0.55 & 0.74 & 0.045 \\
\hline 3 & AM1401-324N & $14: 04: 17.7$ & $-33: 01: 36.4$ & $-19.78 \pm 0.30$ & 0.47 & 0.62 & 0.046 \\
\hline $\mathrm{N}$ & AM1401-324S & $14: 04: 17.9$ & $-33: 02: 9.4$ & $-17.30 \pm 0.30$ & 0.71 & 0.92 & 0.044 \\
\hline 4 & AM1401-324N & $14: 04: 18.0$ & $-33: 01: 31.0$ & $-17.12 \pm 0.30$ & 0.47 & 0.72 & 0.046 \\
\hline 6 & AM1401-324N & $14: 04: 18.1$ & $-33: 01: 19.1$ & $-14.68 \pm 0.30$ & 0.35 & 0.39 & 0.393 \\
\hline 5 & AM1401-324N & $14: 04: 18.7$ & $-33: 01: 38.9$ & $-13.98 \pm 0.30$ & 0.14 & 0.16 & 0.666 \\
\hline 4 & AM1448-262SW & $14: 51: 11.4$ & $-26: 38: 16.2$ & $-13.27 \pm 0.40$ & 0.67 & 1.11 & 0.352 \\
\hline 3 & AM1448-262SW & $14: 51: 11.5$ & $-26: 38: 18.6$ & $-14.12 \pm 0.40$ & 0.75 & 1.25 & 0.362 \\
\hline 1 & AM1448-262SW & $14: 51: 11.7$ & $-26: 38: 14.7$ & $-16.52 \pm 0.40$ & 0.76 & 1.30 & 0.367 \\
\hline 2 & AM1448-262SW & $14: 51: 11.7$ & $-26: 38: 15.4$ & $-15.45 \pm 0.40$ & 0.85 & 1.42 & 0.367 \\
\hline $\mathrm{N}$ & AM1448-262NE & $14: 51: 15.0$ & $-26: 37: 50.5$ & $-15.73 \pm 0.40$ & 1.31 & 1.63 & 0.388 \\
\hline 2 & AM2030-303SW & $20: 33: 55.0$ & $-30: 22: 29.3$ & $-15.60 \pm 0.40$ & 0.61 & 1.02 & 0.348 \\
\hline $1(\mathrm{~N} ?)$ & AM2030-303SW & $20: 33: 55.5$ & $-30: 22: 26.7$ & $-18.77 \pm 0.40$ & 0.53 & 1.05 & 0.372 \\
\hline 4 & AM2030-303NE & $20: 33: 58.0$ & $-30: 22: 13.0$ & $-11.74 \pm 0.40$ & 1.30 & 0.95 & 0.066 \\
\hline 1 & AM2030-303NE & 20:33:59.1 & $-30: 22: 20.3$ & $-17.69 \pm 0.40$ & 0.84 & 1.30 & 0.340 \\
\hline 2 & AM2030-303NE & $20: 33: 59.3$ & $-30: 22: 12.2$ & $-18.61 \pm 0.40$ & 0.60 & 1.15 & 0.372 \\
\hline $3(\mathrm{~N} ?)$ & AM2030-303NE & $20: 33: 59.8$ & $-30: 22: 13.4$ & $-17.98 \pm 0.40$ & 0.61 & 1.17 & 0.372 \\
\hline 4 & AM2058-381N & 21:01:33.7 & $-38: 05: 10.2$ & $-18.14 \pm 0.27$ & 0.33 & 0.65 & 0.344 \\
\hline 3 & AM2058-381N & 21:01:34.0 & $-38: 04: 48.4$ & $-15.40 \pm 0.27$ & 0.31 & 0.62 & 0.344 \\
\hline 2 & AM2058-381N & $21: 01: 34.0$ & $-38: 04: 45.3$ & $-15.35 \pm 0.27$ & 0.41 & 0.47 & 0.468 \\
\hline 11 & AM2058-381N & 21:01:34.1 & $-38: 05: 5.1$ & $-17.10 \pm 0.27$ & 0.58 & 0.86 & 0.045 \\
\hline 1 & AM2058-381N & $21: 01: 34.2$ & $-38: 04: 42.9$ & $-15.13 \pm 0.27$ & 0.32 & 0.67 & 0.352 \\
\hline 10 & AM2058-381N & $21: 01: 34.4$ & $-38: 05: 1.9$ & $-17.56 \pm 0.27$ & 0.58 & 0.87 & 0.033 \\
\hline $\mathrm{N}$ & AM2058-381N & $21: 01: 34.4$ & $-38: 05: 7.0$ & $-18.18 \pm 0.27$ & 0.99 & 1.14 & 0.375 \\
\hline 5 & AM2058-381N & 21:01:34.6 & $-38: 05: 15.6$ & $-17.80 \pm 0.27$ & 0.41 & 0.75 & 0.334 \\
\hline 9 & AM2058-381N & $21: 01: 34.6$ & $-38: 04: 58.5$ & $-17.41 \pm 0.27$ & 0.50 & 0.77 & 0.033 \\
\hline 8 & AM2058-381N & 21:01:34.9 & $-38: 04: 59.3$ & $-17.34 \pm 0.27$ & 0.46 & 0.75 & 0.246 \\
\hline 7 & AM2058-381N & $21: 01: 35.2$ & $-38: 05: 3.9$ & $-17.12 \pm 0.27$ & 0.44 & 0.70 & 0.066 \\
\hline $\mathrm{N}$ & AM2058-381S & $21: 01: 35.2$ & $-38: 06: 0.7$ & $-19.24 \pm 0.27$ & 0.27 & 0.47 & 0.033 \\
\hline 6 & AM2058-381N & $21: 01: 35.3$ & $-38: 05: 18.1$ & $-15.41 \pm 0.27$ & 0.44 & 0.50 & 0.554 \\
\hline $\mathrm{N}$ & AM2105-332NW & 21:08:0.4 & $-33: 12: 3.1$ & $-17.90 \pm 0.30$ & 0.63 & 1.57 & 0.276 \\
\hline $\mathrm{N}$ & AM2105-332SE & 21:08:4.3 & $-33: 13: 17.6$ & $-18.70 \pm 0.30$ & 0.92 & 1.65 & 0.366 \\
\hline 4 & AM2229-735W & $22: 33: 49.7$ & $-73: 40: 20.4$ & $-17.38 \pm 0.40$ & 0.70 & 1.17 & 0.356 \\
\hline 1 & AM2229-735W & $22: 33: 50.6$ & $-73: 40: 25.1$ & $-17.82 \pm 0.40$ & 0.76 & 1.28 & 0.362 \\
\hline 5 & AM2229-735W & $22: 33: 50.9$ & $-73: 40: 28.7$ & $-17.96 \pm 0.40$ & 0.72 & 1.24 & 0.367 \\
\hline 6 & AM2229-735W & $22: 33: 50.9$ & $-73: 40: 36.8$ & $-18.44 \pm 0.40$ & 0.61 & 1.16 & 0.372 \\
\hline $\mathrm{N}$ & AM2229-735W & $22: 33: 51.0$ & $-73: 40: 32.7$ & $-19.49 \pm 0.40$ & 0.66 & 1.21 & 0.372 \\
\hline 2 & AM2229-735W & $22: 33: 52.1$ & $-73: 40: 27.3$ & $-17.31 \pm 0.40$ & 0.55 & 1.06 & 0.372 \\
\hline 3 & AM2229-735W & $22: 33: 54.3$ & $-73: 40: 19.2$ & $-16.18 \pm 0.40$ & 0.71 & 1.18 & 0.356 \\
\hline $\mathrm{N}$ & AM2229-735E & $22: 33: 55.0$ & $-73: 40: 41.0$ & $-18.69 \pm 0.40$ & 0.56 & 1.12 & 0.373 \\
\hline 12 & AM2238-575W & $22: 41: 45.0$ & $-57: 36: 19.5$ & $-15.50 \pm 0.30$ & 1.04 & 1.80 & 0.366 \\
\hline 1 & AM2238-575W & $22: 41: 45.1$ & $-57: 36: 17.1$ & $-16.90 \pm 0.30$ & 1.03 & 1.91 & 0.349 \\
\hline 2 & AM2238-575W & $22: 41: 45.4$ & $-57: 37: 57.9$ & $-15.00 \pm 0.30$ & 0.82 & 1.87 & 0.256 \\
\hline 9 & AM2238-575W & $22: 41: 46.4$ & $-57: 36: 41.1$ & $-16.60 \pm 0.30$ & 0.85 & 1.85 & 0.276 \\
\hline 10 & AM2238-575W & $22: 41: 47.0$ & $-57: 36: 37.9$ & $-16.70 \pm 0.30$ & 0.96 & 1.87 & 0.324 \\
\hline 3 & AM2238-575W & $22: 41: 47.2$ & $-57: 36: 50.0$ & $-17.40 \pm 0.30$ & 0.78 & 1.70 & 0.296 \\
\hline 8 & AM2238-575W & $22: 41: 47.5$ & $-57: 36: 36.5$ & $-16.30 \pm 0.30$ & 0.99 & 1.80 & 0.349 \\
\hline 6 & AM2238-575W & $22: 41: 48.0$ & $-57: 36: 25.6$ & $-17.30 \pm 0.30$ & 1.03 & 1.97 & 0.324 \\
\hline 7 & AM2238-575W & $22: 41: 48.1$ & $-57: 36: 11.2$ & $-15.50 \pm 0.30$ & 0.89 & 1.78 & 0.324 \\
\hline 4 & AM2238-575W & $22: 41: 48.3$ & $-57: 36: 41.9$ & $-15.20 \pm 0.30$ & 0.93 & 1.86 & 0.324 \\
\hline 5 & AM2238-575W & $22: 41: 48.6$ & $-57: 36: 38.3$ & $-15.70 \pm 0.30$ & 0.78 & 1.76 & 0.276 \\
\hline 11 & AM2238-575W & $22: 41: 49.4$ & $-57: 36: 3.7$ & $-14.70 \pm 0.30$ & 0.85 & 1.43 & 0.367 \\
\hline $\mathrm{N}$ & AM2238-575E & $22: 41: 54.8$ & $-57: 36: 8.0$ & $-18.07 \pm 0.30$ & 1.24 & 1.90 & 0.118 \\
\hline 5 & AM2306-721E & 23:09:37.6 & $-72: 01: 33.5$ & $-14.50 \pm 0.27$ & 0.44 & 0.70 & 0.066 \\
\hline
\end{tabular}


D. L. Ferreiro et al.: Modelling HII region properties $(R N)$, Online Material $p 8$

Table A.1. continued.

\begin{tabular}{|c|c|c|c|c|c|c|c|}
\hline $\begin{array}{l}\text { Reg. } \\
\text { No. }\end{array}$ & $\begin{array}{c}\text { Galaxy } \\
\text { Arp }\end{array}$ & $\begin{array}{c}\alpha \\
\mathrm{J} 2000\end{array}$ & $\begin{array}{c}\delta \\
\mathrm{J} 2000\end{array}$ & $\begin{array}{c}M_{B} \\
{[\mathrm{mag}]}\end{array}$ & $\overline{\overline{B-V}}$ & $\overline{\overline{V-I}}$ & $\overline{\overline{E(B-V)}}$ \\
\hline 4 & AM2306-721E & $23: 09: 37.9$ & $-72: 01: 30.4$ & $-14.46 \pm 0.27$ & 0.43 & 0.63 & 0.045 \\
\hline 3 & AM2306-721E & 23:09:38.2 & $-72: 01: 28.4$ & $-14.52 \pm 0.27$ & 0.43 & 0.66 & 0.045 \\
\hline 6 & AM2306-721E & $23: 09: 38.8$ & $-72: 01: 45.8$ & $-17.25 \pm 0.27$ & 0.49 & 0.93 & 0.362 \\
\hline 7 & AM2306-721E & 23:09:40.0 & $-72: 01: 46.2$ & $-16.26 \pm 0.27$ & 0.48 & 0.92 & 0.362 \\
\hline $\mathrm{N}$ & AM2306-721E & 23:09:41.0 & $-72: 01: 38.3$ & $-17.63 \pm 0.27$ & 0.62 & 1.28 & 0.366 \\
\hline 2 & AM2306-721E & 23:09:41.2 & $-72: 01: 31.2$ & $-15.15 \pm 0.27$ & 0.50 & 0.97 & 0.367 \\
\hline 8 & AM2306-721E & 23:09:41.6 & $-72: 01: 47.4$ & $-15.42 \pm 0.27$ & 0.48 & 0.91 & 0.362 \\
\hline 9 & AM2306-721E & 23:09:41.8 & $-72: 01: 44.6$ & $-15.76 \pm 0.27$ & 0.48 & 0.91 & 0.362 \\
\hline 1 & AM2306-721E & 23:09:42.9 & $-72: 01: 29.2$ & $-16.24 \pm 0.27$ & 0.48 & 0.85 & 0.348 \\
\hline 10 & AM2306-721E & $23: 09: 43.4$ & $-72: 01: 40.3$ & $-15.63 \pm 0.27$ & 0.48 & 0.88 & 0.356 \\
\hline 3 & AM2306-721N & $23: 09: 45.4$ & $-72: 00: 3.7$ & $-18.55 \pm 0.27$ & 0.42 & 0.59 & 0.045 \\
\hline 2(N?) & AM2306-721N & 23:09:46.3 & $-72: 00: 9.2$ & $-18.16 \pm 0.27$ & 0.50 & 1.00 & 0.372 \\
\hline 1 & AM2306-721N & 23:09:47.1 & $-72: 00: 12.8$ & $-18.50 \pm 0.27$ & 0.45 & 0.83 & 0.356 \\
\hline 5 & AM2322-821NW & $23: 26: 0.8$ & $-81: 52: 34.2$ & $-13.76 \pm 0.30$ & 0.68 & 0.77 & - \\
\hline 6 & AM2322-821NW & $23: 26: 2.5$ & $-81: 52: 28.7$ & $-14.95 \pm 0.30$ & 0.68 & 0.66 & - \\
\hline 7 & AM2322-821NW & $23: 26: 4.6$ & $-81: 52: 33.1$ & $-14.40 \pm 0.30$ & 0.78 & 0.85 & - \\
\hline 4 & AM2322-821NW & $23: 26: 4.9$ & $-81: 52: 44.5$ & $-14.81 \pm 0.30$ & 1.04 & 1.08 & - \\
\hline 3 & AM2322-821NW & $23: 26: 5.5$ & $-81: 52: 38.8$ & $-14.32 \pm 0.30$ & 0.77 & 0.81 & - \\
\hline 2 & AM2322-821NW & $23: 26: 6.7$ & $-81: 52: 39.8$ & $-14.98 \pm 0.30$ & 0.87 & 0.96 & - \\
\hline $1(\mathrm{~N} ?)$ & AM2322-821NW & $23: 26: 8.3$ & $-81: 52: 40.2$ & $-15.52 \pm 0.30$ & 0.67 & 0.76 & - \\
\hline 26 & AM2322-821SE & $23: 26: 11.8$ & $-81: 54: 45.8$ & $-13.23 \pm 0.30$ & 0.71 & 0.66 & - \\
\hline 27 & AM2322-821SE & $23: 26: 17.4$ & $-81: 55: 11.5$ & $-12.83 \pm 0.30$ & 0.86 & 0.60 & - \\
\hline 25 & AM2322-821SE & $23: 26: 18.2$ & $-81: 53: 58.3$ & $-12.98 \pm 0.30$ & 0.62 & 0.58 & - \\
\hline 24 & AM2322-821SE & $23: 26: 19.4$ & $-81: 53: 55.1$ & $-13.88 \pm 0.30$ & 0.68 & 0.68 & - \\
\hline 23 & AM2322-821SE & $23: 26: 28.0$ & $-81: 53: 45.0$ & $-13.66 \pm 0.30$ & 0.63 & 0.63 & - \\
\hline 22 & AM2322-821SE & $23: 26: 30.2$ & $-81: 53: 47.2$ & $-13.15 \pm 0.30$ & 0.71 & 0.65 & - \\
\hline 21 & AM2322-821SE & $23: 26: 31.7$ & $-81: 53: 43.6$ & $-13.52 \pm 0.30$ & 0.73 & 0.60 & - \\
\hline 4 & AM2322-821SE & $23: 26: 32.1$ & $-81: 54: 40.5$ & $-14.89 \pm 0.30$ & 0.92 & 0.88 & - \\
\hline 5 & AM2322-821SE & $23: 26: 32.5$ & $-81: 54: 44.0$ & $-14.38 \pm 0.30$ & 0.93 & 0.89 & - \\
\hline 3 & AM2322-821SE & $23: 26: 33.1$ & $-81: 54: 37.3$ & $-13.61 \pm 0.30$ & 1.06 & 1.10 & - \\
\hline 2 & AM2322-821SE & $23: 26: 33.4$ & $-81: 54: 34.5$ & $-14.24 \pm 0.30$ & 1.07 & 1.05 & - \\
\hline 6 & AM2322-821SE & $23: 26: 33.6$ & $-81: 54: 46.8$ & $-13.88 \pm 0.30$ & 0.89 & 0.85 & - \\
\hline 8 & AM2322-821SE & $23: 26: 34.2$ & $-81: 54: 58.3$ & $-13.37 \pm 0.30$ & 0.95 & 0.84 & - \\
\hline 7 & AM2322-821SE & $23: 26: 34.7$ & $-81: 55: 0.5$ & $-13.37 \pm 0.30$ & 0.86 & 0.84 & - \\
\hline 1 & AM2322-821SE & $23: 26: 35.0$ & $-81: 54: 35.1$ & $-14.74 \pm 0.30$ & 1.15 & 1.20 & - \\
\hline 10 & AM2322-821SE & $23: 26: 35.7$ & $-81: 54: 50.8$ & $-14.44 \pm 0.30$ & 0.97 & 0.94 & - \\
\hline 9 & AM2322-821SE & $23: 26: 36.3$ & $-81: 55: 2.8$ & $-12.69 \pm 0.30$ & 0.82 & 0.74 & - \\
\hline 20 & AM2322-821SE & $23: 26: 36.4$ & $-81: 54: 24.4$ & $-14.67 \pm 0.30$ & 0.99 & 1.03 & - \\
\hline 19 & AM2322-821SE & $23: 26: 37.0$ & $-81: 54: 1.6$ & $-14.68 \pm 0.30$ & 0.87 & 0.82 & - \\
\hline $\mathrm{N}$ & AM2322-821SE & $23: 26: 37.1$ & $-81: 54: 38.1$ & $-16.99 \pm 0.30$ & 1.18 & 1.22 & - \\
\hline 17 & AM2322-821SE & $23: 26: 41.2$ & $-81: 54: 17.7$ & $-13.19 \pm 0.30$ & 0.96 & 0.89 & - \\
\hline 11 & AM2322-821SE & $23: 26: 41.6$ & $-81: 54: 49.6$ & $-14.41 \pm 0.30$ & 0.95 & 0.89 & - \\
\hline 16 & AM2322-821SE & $23: 26: 43.5$ & $-81: 54: 26.4$ & $-13.39 \pm 0.30$ & 0.98 & 0.99 & - \\
\hline 15 & AM2322-821SE & $23: 26: 43.9$ & $-81: 54: 30.9$ & $-14.01 \pm 0.30$ & 0.93 & 0.86 & - \\
\hline 14 & AM2322-821SE & $23: 26: 44.7$ & $-81: 54: 36.5$ & $-14.39 \pm 0.30$ & 0.91 & 0.87 & - \\
\hline 12 & AM2322-821SE & $23: 26: 45.4$ & $-81: 54: 44.4$ & $-14.58 \pm 0.30$ & 0.93 & 0.87 & - \\
\hline 18 & AM2322-821SE & $23: 26: 45.5$ & $-81: 54: 9.8$ & $-13.04 \pm 0.30$ & 0.84 & 0.84 & - \\
\hline 13 & AM2322-821SE & $23: 26: 51.4$ & $-81: 54: 40.2$ & $-14.81 \pm 0.30$ & 0.74 & 0.71 & - \\
\hline 3 & AM2330-451SW & $23: 33: 18.4$ & $-45: 01: 18.8$ & $-14.88 \pm 0.30$ & 0.74 & 1.04 & 0.045 \\
\hline 1 & AM2330-451SW & $23: 33: 18.6$ & $-45: 01: 6.9$ & $-13.87 \pm 0.30$ & 0.84 & 1.12 & 0.045 \\
\hline $\mathrm{N}$ & AM2330-451NE & $23: 33: 21.1$ & $-45: 00: 43.7$ & $-17.90 \pm 0.30$ & 0.80 & 1.48 & 0.373 \\
\hline
\end{tabular}

Table A.2. HII region properties in a sample of minor merger II: Region, Arp galaxy, luminosity $H \alpha$, equivalent with, star formation rates, age, and mass.

\begin{tabular}{|c|c|c|c|c|c|c|}
\hline Reg. & 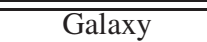 & $\overline{\overline{\mathrm{L}(\mathrm{H} \alpha)}}$ & $\overline{E E W(\dagger)}$ & $\overline{\overline{\text { Age }}}$ & $\overline{S F R(\mathrm{H} \alpha)}$ & $\overline{\text { Mass }}$ \\
\hline No. & Arp & {$\left[\operatorname{erg~s}^{-1}\right]$} & \pm 0.1 & [Myr] & {$\left[M_{\odot} \mathrm{yr}^{-1}\right]$} & {$\left[M_{\odot}\right]$} \\
\hline 7 & AM1256-433W & $1.247 \times 10^{40} \pm 2.41 \%$ & 2.0 & $5.81 \pm 0.14$ & $0.099 \pm 0.002$ & $5 \times 10^{6} \pm 1 \times 10^{6}$ \\
\hline 6 & AM1256-433W & $1.123 \times 10^{41} \pm 2.07 \%$ & 1.9 & $5.95 \pm 0.16$ & $0.89 \pm 0.02$ & $5 \times 10^{7} \pm 1 \times 10^{6}$ \\
\hline $5(\mathrm{~N} ?)$ & AM1256-433W & $1.040 \times 10^{41} \pm 2.21 \%$ & 2.0 & $5.81 \pm 0.14$ & $0.82 \pm 0.02$ & $5 \times 10^{7} \pm 1 \times 10^{6}$ \\
\hline 4 & AM1256-433W & $4.120 \times 10^{40} \pm 2.22 \%$ & 1.9 & $5.95 \pm 0.16$ & $0.325 \pm 0.007$ & $3 \times 10^{7} \pm 6 \times 10^{6}$ \\
\hline 3 & AM1256-433W & $3.103 \times 10^{40} \pm 2.59 \%$ & 2.0 & $5.81 \pm 0.14$ & $0.245 \pm 0.006$ & $1 \times 10^{7} \pm 7 \times 10^{6}$ \\
\hline 2 & AM1256-433W & $3.722 \times 10^{40} \pm 2.66 \%$ & 2.1 & $5.66 \pm 0.16$ & $0.294 \pm 0.008$ & $1 \times 10^{7} \pm 3 \times 10^{6}$ \\
\hline 1 & AM1256-433W & $1.930 \times 10^{40} \pm 2.38 \%$ & 1.9 & $5.95 \pm 0.16$ & $0.152 \pm 0.004$ & $1 \times 10^{7} \pm 4 \times 10^{4}$ \\
\hline 2 & AM1256-433E & - & 1.4 & $6.96 \pm 0.31$ & ${ }^{(\star)} 0.036 \pm 0.005$ & ${ }^{(*)} 5 \times 10^{6} \pm 5 \times 10^{5}$ \\
\hline
\end{tabular}


Table A.2. continued.

\begin{tabular}{|c|c|c|c|c|c|c|}
\hline $\begin{array}{l}\text { Reg. } \\
\text { No. }\end{array}$ & $\begin{array}{l}\text { Galaxy } \\
\text { Arp }\end{array}$ & $\begin{array}{c}\mathcal{L}(\mathrm{H} \alpha) \\
{\left[\operatorname{erg~s}^{-1}\right]}\end{array}$ & $\begin{array}{c}E W(\dagger) \\
\pm 0.1 \\
\end{array}$ & $\begin{array}{c}\text { Age } \\
{[\mathrm{Myr}]}\end{array}$ & $\begin{array}{l}S F R(\mathrm{H} \alpha) \\
{\left[M_{\odot} \mathrm{yr}^{-1}\right]}\end{array}$ & $\begin{array}{l}\text { Mass } \\
{\left[M_{\odot}\right]}\end{array}$ \\
\hline 1 & AM1256-433E & - & 0.6 & $13.0 \pm 0.5$ & 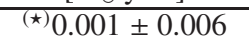 & (*) $3 \times 10^{8} \pm 4 \times 10^{7}$ \\
\hline 2 & AM1401-324N & $2.461 \times 10^{41} \pm 1.96 \%$ & 2.0 & $5.81 \pm 0.14$ & $1.94 \pm 0.04$ & $1 \times 10^{8} \pm 2 \times 10^{7}$ \\
\hline 1 & AM1401-324N & $4.263 \times 10^{40} \pm 2.30 \%$ & 1.9 & $5.95 \pm 0.16$ & $0.337 \pm 0.008$ & $3 \times 10^{7} \pm 6 \times 10^{6}$ \\
\hline $\mathrm{N}$ & AM1401-324N & $1.999 \times 10^{41} \pm 1.95 \%$ & 1.1 & $10.2 \pm 0.7$ & $1.58 \pm 0.03$ & $1 \times 10^{9} \pm 3 \times 10^{8}$ \\
\hline 3 & AM1401-324N & $1.457 \times 10^{41} \pm 1.93 \%$ & 1.1 & $10.2 \pm 0.7$ & $1.15 \pm 0.02$ & $1 \times 10^{9} \pm 9 \times 10^{7}$ \\
\hline $\mathrm{N}$ & AM1401-324S & $1.167 \times 10^{40} \pm 4.66 \%$ & 1.3 & $7.38 \pm 0.46$ & $0.092 \pm 0.004$ & $3 \times 10^{7} \pm 1 \times 10^{7}$ \\
\hline 4 & AM1401-324N & $3.940 \times 10^{40} \pm 2.02 \%$ & 1.6 & $6.50 \pm 0.19$ & $0.311 \pm 0.006$ & $3 \times 10^{7} \pm \times 10$ \\
\hline 6 & AM1401-324N & $1.162 \times 10^{40} \pm 6.83 \%$ & 2.1 & $5.66 \pm 0.16$ & $0.092 \pm 0.006$ & $5 \times 10^{6} \pm 9 \times 10^{5}$ \\
\hline 5 & AM1401-324N & $1.151 \times 10^{40} \pm 2.81 \%$ & 2.3 & $5.29 \pm 0.13$ & $0.091 \pm 0.003$ & $3 \times 10^{6} \pm 4 \times 10^{5}$ \\
\hline 4 & AM1448-262SW & - & 1.6 & $6.50 \pm 0.19$ & ${ }^{(\star)} 0.003 \pm 0.006$ & (*) $3 \times 10^{5} \pm 9 \times 10^{4}$ \\
\hline 3 & AM1448-262SW & - & 1.7 & $6.31 \pm 0.19$ & $(\star) 0.004 \pm 0.005$ & (*) $3 \times 10^{5} \pm 9 \times 10^{4}$ \\
\hline 1 & AM1448-262SW & - & 1.6 & $6.50 \pm 0.19$ & ${ }^{(\star)} 0.031 \pm 0.006$ & (*) $3 \times 10^{6} \pm 7 \times 10^{5}$ \\
\hline 2 & AM1448-262SW & - & 1.8 & $6.13 \pm 0.17$ & ${ }^{(\star)} 0.014 \pm 0.006$ & (*) $1 \times 10^{6} \pm 5 \times 10^{5}$ \\
\hline $\mathrm{N}$ & AM1448-262NE & - & 0.5 & $13.5 \pm 0.6$ & ${ }^{(\star)} 0.003 \pm 0.005$ & (*) $1 \times 10^{7} \pm 2 \times 10^{6}$ \\
\hline 2 & AM2030-303SW & $3.276 \times 10^{40} \pm 2.47 \%$ & 1.9 & $5.95 \pm 0.16$ & $0.259 \pm 0.007$ & $1 \times 10^{7} \pm 1 \times 10^{7}$ \\
\hline $1(\mathrm{~N} ?)$ & AM2030-303SW & $2.715 \times 10^{41} \pm 2.84 \%$ & 1.9 & $5.95 \pm 0.16$ & $2.14 \pm 0.05$ & $1 \times 10^{8} \pm 6 \times 10^{7}$ \\
\hline 4 & AM2030-303NE & $1.359 \times 10^{40} \pm 4.96 \%$ & 2.7 & $4.22 \pm 0.28$ & $0.107 \pm 0.005$ & (*) $1 \times 10^{6} \pm 2 \times 10^{5}$ \\
\hline 1 & AM2030-303NE & $8.652 \times 10^{38} \pm 2.55 \%$ & 1.7 & 6.310 .19 & $0.0068 \pm 0.0002$ & $5 \times 10^{5} \pm 4 \times 10^{5}$ \\
\hline 2 & AM2030-303NE & $5.630 \times 10^{39} \pm 2.39 \%$ & 2.1 & $5.66 \pm 0.16$ & $0.044 \pm 0.001$ & $3 \times 10^{6} \pm 9 \times 10^{5}$ \\
\hline 3(N?) & AM2030-303NE & $3.464 \times 10^{40} \pm 2.42 \%$ & 1.7 & $6.31 \pm 0.19$ & $0.274 \pm 0.007$ & $3 \times 10^{7} 5 \pm \times 10^{6}$ \\
\hline 4 & AM2058-381N & $4.652 \times 10^{39} \pm 2.91 \%$ & 2.0 & $5.81 \pm 0.14$ & $0.037 \pm 0.001$ & $3 \times 10^{6} \pm 6 \times 10^{5}$ \\
\hline 3 & AM2058-381N & $5.054 \times 10^{39} \pm 2.89 \%$ & 2.2 & $5.44 \pm 0.21$ & $0.040 \pm 0.001$ & $1 \times 10^{6} \pm 8 \times 10^{5}$ \\
\hline 2 & AM2058-381N & $8.135 \times 10^{39} \pm 3.41 \%$ & 2.4 & $5.15 \pm 0.16$ & $0.064 \pm 0.002$ & $1 \times 10^{6} \pm 7 \times 10^{5}$ \\
\hline 11 & AM2058-381N & $9.208 \times 10^{39} \pm 3.09 \%$ & 1.5 & $6.70 \pm 0.21$ & $0.073 \pm 0.002$ & $1 \times 10^{7} \pm 2 \times 10^{6}$ \\
\hline 1 & AM2058-381N & $5.042 \times 10^{39} \pm 3.33 \%$ & 2.2 & $5.44 \pm 0.21$ & $0.040 \pm 0.001$ & $1 \times 10^{6} \pm 8 \times 10^{5}$ \\
\hline 10 & AM2058-381N & $2.244 \times 10^{40} \pm 3.19 \%$ & 1.6 & $6.50 \pm 0.19$ & $0.177 \pm 0.006$ & (*) $1 \times 10^{7} \pm 9 \times 10^{6}$ \\
\hline $\mathrm{N}$ & AM2058-381N & $5.872 \times 10^{40} \pm 3.26 \%$ & 1.1 & $10.2 \pm 0.7$ & $0.46 \pm 0.02$ & $5 \times 10^{8} \pm 2 \times 10^{7}$ \\
\hline 5 & AM2058-381N & $3.412 \times 10^{40} \pm 3.74 \%$ & 1.9 & $5.95 \pm 0.16$ & $0.27 \pm 0.01$ & $3 \times 10^{7} \pm 1 \times 10^{7}$ \\
\hline 9 & AM2058-381N & $1.921 \times 10^{40} \pm 2.88 \%$ & 1.7 & $6.31 \pm 0.19$ & $0.152 \pm 0.004$ & $1 \times 10^{7} \pm 4 \times 10^{6}$ \\
\hline 8 & AM2058-381N & $1.572 \times 10^{40} \pm 2.95 \%$ & 1.7 & $6.31 \pm 0.19$ & $0.124 \pm 0.004$ & $1 \times 10^{7} \pm 5 \times 10^{5}$ \\
\hline 7 & AM2058-381N & $2.066 \times 10^{40} \pm 3.10 \%$ & 1.9 & $5.95 \pm 0.16$ & $0.163 \pm 0.005$ & $1 \times 10^{7} \pm 1 \times 10^{6}$ \\
\hline $\mathrm{N}$ & AM2058-381S & $9.392 \times 10^{40} \pm 2.84 \%$ & 1.6 & $6.50 \pm 0.19$ & $0.74 \pm 0.02$ & $1 \times 10^{8} \pm 2 \times 10^{7}$ \\
\hline 6 & AM2058-381N & $6.523 \times 10^{39} \pm 3.10 \%$ & 2.1 & $5.66 \pm 0.16$ & $0.052 \pm 0.002$ & $3 \times 10^{6} \pm 6 \times 10^{5}$ \\
\hline $\mathrm{N}$ & AM2105-332NW & $1.322 \times 10^{39} \pm 2.21 \%$ & 1.6 & $6.50 \pm 0.19$ & $0.0104 \pm 0.0002$ & $1 \times 10^{6} \pm \times 10$ \\
\hline $\mathrm{N}$ & AM2105-332SE & $2.405 \times 10^{38} \pm 1.73 \%$ & 0.6 & $13.0 \pm 0.5$ & $0.00190 \pm 0.00003$ & $5 \times 10^{6} \pm 3 \times 10^{5}$ \\
\hline 4 & AM2229-735W & $2.096 \times 10^{41} \pm 0.94 \%$ & 1.9 & $5.95 \pm 0.16$ & $1.66 \pm 0.02$ & $1 \times 10^{8} \pm 1 \times 10^{7}$ \\
\hline 1 & AM2229-735W & $2.824 \times 10^{41} \pm 0.75 \%$ & 1.8 & $6.13 \pm 0.17$ & $2.23 \pm 0.02$ & $1 \times 10^{8} \pm 9 \times 10^{7}$ \\
\hline 5 & AM2229-735W & $2.768 \times 10^{41} \pm 4.91 \%$ & 1.8 & $6.13 \pm 0.17$ & $2.2 \pm 0.1$ & $1 \times 10^{8} \pm 9 \times 10^{7}$ \\
\hline 6 & AM2229-735W & $1.552 \times 10^{41} \pm 0.90 \%$ & 1.6 & $6.50 \pm 0.19$ & $1.23 \pm 0.01$ & $1 \times 10^{8} \pm 2 \times 10^{7}$ \\
\hline $\mathrm{N}$ & AM2229-735W & $8.081 \times 10^{41} \pm 1.14 \%$ & 1.8 & $6.13 \pm 0.17$ & $6.38 \pm 0.07$ & $5 \times 10^{8} \pm 6 \times 10^{6}$ \\
\hline 2 & AM2229-735W & $3.559 \times 10^{41} \pm 0.78 \%$ & 2.4 & $5.15 \pm 0.16$ & $2.81 \pm 0.02$ & $1 \times 10^{8} \pm 2 \times 10^{7}$ \\
\hline 3 & AM2229-735W & $1.355 \times 10^{40} \pm 0.83 \%$ & 1.5 & $6.70 \pm 0.21$ & $0.1071 \pm 0.0009$ & $1 \times 10^{7} \pm 2 \times 10^{6}$ \\
\hline $\mathrm{N}$ & AM2229-735E & $6.909 \times 10^{41} \pm 1.50 \%$ & 2.1 & $5.66 \pm 0.16$ & $5.46 \pm 0.08$ & $3 \times 10^{8} \pm \times 10$ \\
\hline 12 & AM2238-575W & - & 1.7 & $6.31 \pm 0.19$ & ${ }^{(\star)} 0.012 \pm 0.005$ & (*) $1 \times 10^{6} \pm 6 \times 10^{5}$ \\
\hline 1 & AM2238-575W & - & 1.7 & $6.31 \pm 0.19$ & $\left(\star{ }^{\star}\right) 0.061 \pm 0.006$ & (*) $5 \times 10^{6} \pm 4 \times 10^{4}$ \\
\hline 2 & AM2238-575W & - & 1.9 & $5.95 \pm 0.16$ & ${ }^{(\star)} 0.016 \pm 0.006$ & (*) $1 \times 10^{6} \pm 6 \times 10^{4}$ \\
\hline 9 & AM2238-575W & - & 1.8 & $6.13 \pm 0.17$ & ${ }^{(\star)} 0.042 \pm 0.007$ & (*) $3 \times 10^{6} \pm 1 \times 10^{6}$ \\
\hline 10 & AM2238-575W & - & 1.8 & $6.13 \pm 0.17$ & 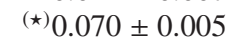 & (*) $5 \times 10^{6} \pm 7 \times 10^{5}$ \\
\hline 3 & AM2238-575W & - & 2.1 & $5.66 \pm 0.16$ & 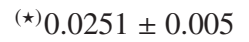 & (*) $1 \times 10^{7} \pm 3 \times 10^{5}$ \\
\hline 8 & AM2238-575W & - & 1.9 & $5.95 \pm 0.16$ & $\left(\star{ }^{\star}\right) 0.049 \pm 0.007$ & (*) $3 \times 10^{6} \pm 2 \times 10^{6}$ \\
\hline 6 & AM2238-575W & - & 1.9 & $5.95 \pm 0.16$ & ${ }^{(\star)} 0.163 \pm 0.006$ & (*) $1 \times 10^{7} \pm 2 \times 10^{6}$ \\
\hline 7 & AM2238-575W & - & 1.9 & $5.95 \pm 0.16$ & ${ }^{(\star)} 0.016 \pm 0.006$ & (*) $1 \times 10^{6} \pm 1 \times 10^{6}$ \\
\hline 4 & AM2238-575W & - & 2.1 & $5.66 \pm 0.16$ & ${ }^{(\star)} 0.025 \pm 0.005$ & (*) $1 \times 10^{6} \pm 1 \times 10^{6}$ \\
\hline 5 & AM2238-575W & - & 1.9 & $5.95 \pm 0.16$ & 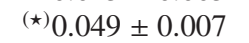 & (*) $3 \times 10^{6} \pm 1 \times 10^{6}$ \\
\hline 11 & AM2238-575W & - & 1.8 & $6.13 \pm 0.17$ & 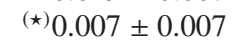 & (*) $5 \times 10^{5} \pm 2 \times 10^{5}$ \\
\hline $\mathrm{N}$ & AM2238-575E & - & 0.7 & $12.6 \pm 0.30$ & 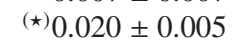 & (*) $5 \times 10^{7} \pm 3 \times 10^{7}$ \\
\hline 5 & AM2306-721E & $8.501 \times 10^{39} \pm 2.44 \%$ & 1.9 & $5.95 \pm 0.16$ & $0.067 \pm 0.002$ & $5 \times 10^{6} \pm 5 \times 10^{5}$ \\
\hline 4 & AM2306-721E & $9.154 \times 10^{39} \pm 3.15 \%$ & 2.0 & $5.81 \pm 0.14$ & $0.072 \pm 0.002$ & $5 \times 10^{6} \pm 6 \times 10^{5}$ \\
\hline 3 & AM2306-721E & $8.303 \times 10^{39} \pm 2.90 \%$ & 1.9 & $5.95 \pm 0.16$ & $0.066 \pm 0.002$ & $5 \times 10^{6} \pm 6 \times 10^{5}$ \\
\hline 6 & AM2306-721E & $1.145 \times 10^{41} \pm 2.12 \%$ & 1.8 & $6.13 \pm 0.17$ & $0.90 \pm 0.02$ & $5 \times 10^{7} \pm 2 \times 10^{7}$ \\
\hline 7 & AM2306-721E & $3.392 \times 10^{40} \pm 2.31 \%$ & 1.7 & $6.31 \pm 0.19$ & $0.268 \pm 0.006$ & $3 \times 10^{7} \pm 5 \times 10^{6}$ \\
\hline $\mathrm{N}$ & AM2306-721E & $7.482 \times 10^{40} \pm 2.27 \%$ & 1.4 & $6.96 \pm 0.31$ & $0.59 \pm 0.01$ & $1 \times 10^{8} \pm 2 \times 10^{7}$ \\
\hline 2 & AM2306-721E & $1.243 \times 10^{40} \pm 2.97 \%$ & 1.7 & $6.31 \pm 0.19$ & $0.098 \pm 0.003$ & $1 \times 10^{7} \pm 1 \times 10^{6}$ \\
\hline 8 & AM2306-721E & $2.744 \times 10^{40} \pm 2.20 \%$ & 1.9 & $5.95 \pm 0.16$ & $0.217 \pm 0.005$ & $1 \times 10^{7} \pm 6 \times 10^{6}$ \\
\hline
\end{tabular}


Table A.2. continued.

\begin{tabular}{|c|c|c|c|c|c|c|}
\hline $\begin{array}{l}\text { Reg. } \\
\text { No. }\end{array}$ & $\begin{array}{c}\text { Galaxy } \\
\text { Arp }\end{array}$ & $\begin{array}{c}\mathcal{L}(\mathrm{H} \alpha) \\
{\left[\operatorname{erg~s}^{-1}\right]}\end{array}$ & $\begin{array}{c}E W(\dagger) \\
\pm 0.1\end{array}$ & $\begin{array}{c}\text { Age } \\
{[\mathrm{Myr}]}\end{array}$ & $\begin{array}{l}S F R(\mathrm{H} \alpha) \\
{\left[M_{\odot} \mathrm{yr}^{-1}\right]}\end{array}$ & $\begin{array}{l}\text { Mass } \\
{\left[M_{\odot}\right]}\end{array}$ \\
\hline 9 & AM2306-721E & $2.438 \times 10^{40} \pm 2.32 \%$ & 1.8 & $6.13 \pm 0.17$ & $0.193 \pm 0.004$ & $3 \times 10^{7} \pm 6 \times 10^{6}$ \\
\hline 1 & AM2306-721E & $5.064 \times 10^{40} \pm 3.06 \%$ & 1.9 & $5.95 \pm 0.16$ & $0.400 \pm 0.009$ & $3 \times 10^{7} \pm 2 \times 10^{6}$ \\
\hline 10 & AM2306-721E & $1.117 \times 10^{40} \pm 2.44 \%$ & 1.6 & $6.50 \pm 0.19$ & $0.088 \pm 0.002$ & $1 \times 10^{7} \pm 1 \times 10^{6}$ \\
\hline 3 & AM2306-721N & $1.319 \times 10^{42} \pm 3.04 \%$ & 2.0 & $5.81 \pm 0.14$ & $10.4 \pm 0.3$ & $5 \times 10^{8} \pm 1 \times 10^{8}$ \\
\hline $2(\mathrm{~N} ?)$ & AM2306-721N & $7.489 \times 10^{41} \pm 3.05 \%$ & 1.8 & $6.13 \pm 0.17$ & $5.9 \pm 0.2$ & $5 \times 10^{8} \pm 4 \times 10^{7}$ \\
\hline 1 & AM2306-721N & $1.272 \times 10^{42} \pm 3.06 \%$ & 1.9 & $5.95 \pm 0.16$ & $10.0 \pm 0.3$ & (*) $5 \times 10^{8} 2 \pm \times 10^{8}$ \\
\hline 5 & AM2322-821NW & $6.543 \times 10^{39} \pm 2.46 \%$ & 1.7 & $6.31 \pm 0.19$ & $0.052 \pm 0.001$ & (*) $^{*} \times 10^{6} \pm 6 \times 10^{5}$ \\
\hline 6 & AM2322-821NW & $1.289 \times 10^{40} \pm 2.24 \%$ & 2.3 & $5.29 \pm 0.13$ & $0.102 \pm 0.002$ & ${ }^{(*)} 3 \times 10^{6} \pm 2 \times 10^{9}$ \\
\hline 7 & AM2322-821NW & $8.336 \times 10^{39} \pm 2.45 \%$ & 2.0 & $5.81 \pm 0.14$ & $0.066 \pm 0.002$ & (*) $^{*} \times 10^{6} \pm 1 \times 10^{6}$ \\
\hline 4 & AM2322-821NW & $1.211 \times 10^{40} \pm 2.26 \%$ & 1.7 & $6.31 \pm 0.19$ & $0.096 \pm 0.002$ & (*) $1 \times 10^{7} \pm 2 \times 10^{6}$ \\
\hline 3 & AM2322-821NW & $1.120 \times 10^{40} \pm 2.29 \%$ & 1.6 & $6.50 \pm 0.19$ & $0.088 \pm 0.002$ & (*) $1 \times 10^{7} \pm 1 \times 10^{6}$ \\
\hline 2 & AM2322-821NW & $1.997 \times 10^{40} \pm 2.40 \%$ & 1.6 & $6.50 \pm 0.19$ & $0.158 \pm 0.004$ & (*) $1 \times 10^{7} \pm 7 \times 10^{6}$ \\
\hline $1(\mathrm{~N} ?)$ & AM2322-821NW & $2.368 \times 10^{40} \pm 2.29 \%$ & 1.3 & $7.38 \pm 0.46$ & $0.187 \pm 0.004$ & (*) $3 \times 10^{7} \pm 7 \times 10^{6}$ \\
\hline 26 & AM2322-821SE & $7.412 \times 10^{39} \pm 3.14 \%$ & 2.3 & $5.29 \pm 0.13$ & $0.059 \pm 0.002$ & (*) $3 \times 10^{6} \pm 8 \times 10^{5}$ \\
\hline 27 & AM2322-821SE & $1.125 \times 10^{40} \pm 3.42 \%$ & 2.4 & $5.15 \pm 0.16$ & $0.089 \pm 0.003$ & ${ }^{(*)} 3 \times 10^{6} \pm 1 \times 10^{6}$ \\
\hline 25 & AM2322-821SE & $2.529 \times 10^{39} \pm 3.16 \%$ & 2.1 & $5.66 \pm 0.16$ & $0.0200 \pm 0.0006$ & ${ }^{(*)} 1 \times 10^{6} \pm 1 \times 10^{5}$ \\
\hline 24 & AM2322-821SE & $9.044 \times 10^{39} \pm 3.28 \%$ & 2.2 & $5.44 \pm 0.21$ & $0.071 \pm 0.002$ & (*) $3 \times 10^{6} \pm 1 \times 10^{5}$ \\
\hline 23 & AM2322-821SE & $9.715 \times 10^{39} \pm 3.18 \%$ & 2.4 & $5.15 \pm 0.16$ & $0.077 \pm 0.002$ & (*) $3 \times 10^{6} \pm 1 \times 10^{6}$ \\
\hline 22 & AM2322-821SE & $6.618 \times 10^{39} \pm 3.43 \%$ & 2.3 & $5.29 \pm 0.13$ & $0.052 \pm 0.002$ & ${ }^{(*)} 1 \times 10^{6} \pm 6 \times 10^{5}$ \\
\hline 21 & AM2322-821SE & $1.139 \times 10^{40} \pm 3.12 \%$ & 2.4 & $5.15 \pm 0.16$ & $0.090 \pm 0.003$ & (*) $3 \times 10^{6} \pm 7 \times 10^{5}$ \\
\hline 4 & AM2322-821SE & $1.534 \times 10^{40} \pm 3.12 \%$ & 1.7 & $6.31 \pm 0.19$ & $0.121 \pm 0.004$ & (*) $1 \times 10^{7} \pm 7 \times 10^{4}$ \\
\hline 5 & AM2322-821SE & $9.521 \times 10^{39} \pm 3.15 \%$ & 1.7 & $6.31 \pm 0.19$ & $0.075 \pm 0.002$ & (*) $5 \times 10^{6} \pm 2 \times 10^{6}$ \\
\hline 3 & AM2322-821SE & $8.071 \times 10^{39} \pm 3.63 \%$ & 1.7 & $6.31 \pm 0.19$ & $0.064 \pm 0.002$ & (*) $5 \times 10^{6} \pm 4 \times 10^{5}$ \\
\hline 2 & AM2322-821SE & $1.959 \times 10^{40} \pm 3.20 \%$ & 1.8 & $6.13 \pm 0.17$ & $0.155 \pm 0.005$ & (*) $1 \times 10^{7} \pm 2 \times 10^{6}$ \\
\hline 6 & AM2322-821SE & $8.321 \times 10^{39} \pm 3.38 \%$ & 1.7 & $6.31 \pm 0.19$ & $0.066 \pm 0.002$ & (*) $^{*} \times 10^{6} \pm 6 \times 10^{5}$ \\
\hline 8 & AM2322-821SE & $1.144 \times 10^{40} \pm 6.12 \%$ & 2.1 & $5.66 \pm 0.16$ & $0.090 \pm 0.006$ & (*) $5 \times 10^{6} \pm 8 \times 10^{5}$ \\
\hline 7 & AM2322-821SE & $8.891 \times 10^{39} \pm 3.43 \%$ & 2.1 & $5.66 \pm 0.16$ & $0.070 \pm 0.002$ & (*) $3 \times 10^{6} \pm 7 \times 10^{4}$ \\
\hline 1 & AM2322-821SE & $2.322 \times 10^{40} \pm 3.16 \%$ & 1.6 & $6.50 \pm 0.19$ & $0.183 \pm 0.006$ & (*) $3 \times 10^{7} \pm 1 \times 10^{7}$ \\
\hline 10 & AM2322-821SE & $1.028 \times 10^{40} \pm 3.20 \%$ & 1.7 & $6.31 \pm 0.19$ & $0.081 \pm 0.003$ & (*) $1 \times 10^{7} \pm 2 \times 10^{6}$ \\
\hline 9 & AM2322-821SE & $5.130 \times 10^{39} \pm 3.16 \%$ & 2.2 & $5.44 \pm 0.21$ & $0.041 \pm 0.001$ & (*) $1 \times 10^{6} \pm 8 \times 10^{5}$ \\
\hline 20 & AM2322-821SE & $1.616 \times 10^{40} \pm 3.32 \%$ & 1.7 & $6.31 \pm 0.19$ & $0.128 \pm 0.002$ & ${ }^{(*)} 1 \times 10^{7} \pm 1 \times 10^{6}$ \\
\hline 19 & AM2322-821SE & $9.432 \times 10^{39} \pm 3.24 \%$ & 1.8 & $6.13 \pm 0.17$ & $0.075 \pm 0.004$ & (*) $5 \times 10^{6} \pm 4 \times 10^{5}$ \\
\hline $\mathrm{N}$ & AM2322-821SE & $1.454 \times 10^{41} \pm 3.15 \%$ & 1.5 & $6.70 \pm 0.21$ & $1.15 \pm 0.04$ & (*) $^{*} \times 10^{8} \pm 3 \times 10^{7}$ \\
\hline 17 & AM2322-821SE & $6.909 \times 10^{39} \pm 3.12 \%$ & 2.0 & $5.81 \pm 0.14$ & $0.055 \pm 0.002$ & ${ }^{(*)} 3 \times 10^{6} \pm 3 \times 10^{5}$ \\
\hline 11 & AM2322-821SE & $1.538 \times 10^{40} \pm 3.11 \%$ & 1.8 & $6.13 \pm 0.17$ & $0.121 \pm 0.004$ & ${ }^{(*)} 1 \times 10^{7} \pm 5 \times 10^{5}$ \\
\hline 16 & AM2322-821SE & $7.114 \times 10^{39} \pm 3.38 \%$ & 1.8 & $6.13 \pm 0.17$ & $0.056 \pm 0.002$ & (*) $5 \times 10^{6} \pm 7 \times 10^{5}$ \\
\hline 15 & AM2322-821SE & $1.117 \times 10^{40} \pm 3.46 \%$ & 1.9 & $5.95 \pm 0.16$ & $0.088 \pm 0.003$ & 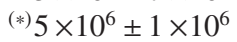 \\
\hline 14 & AM2322-821SE & $1.163 \times 10^{40} \pm 3.42 \%$ & 1.8 & $6.13 \pm 0.17$ & $0.092 \pm 0.003$ & (*) $1 \times 10^{7} \pm 2 \times 10^{6}$ \\
\hline 12 & AM2322-821SE & $1.207 \times 10^{40} \pm 3.16 \%$ & 2.0 & $5.81 \pm 0.14$ & $0.095 \pm 0.002$ & (*) $5 \times 10^{6} \pm 9 \times 10^{5}$ \\
\hline 18 & AM2322-821SE & $6.645 \times 10^{39} \pm 3.14 \%$ & 2.1 & $5.66 \pm 0.16$ & $0.052 \pm 0.003$ & (*) $3 \times 10^{6} \pm 6 \times 10^{5}$ \\
\hline 13 & AM2322-821SE & $1.921 \times 10^{40} \pm 3.14 \%$ & 2.1 & $5.66 \pm 0.16$ & $0.152 \pm 0.005$ & (*) $^{*} \times 10^{6} \pm 1 \times 10^{6}$ \\
\hline 3 & AM2330-451SW & - & 1.5 & $6.70 \pm 0.21$ & ${ }^{(\star)} 0.009 \pm 0.006$ & ${ }^{(*)} 1 \times 10^{6} \pm 1 \times 10^{5}$ \\
\hline 1 & AM2330-451SW & - & 1.5 & $6.70 \pm 0.21$ & $\left.{ }^{\star \star}\right) 0.003 \pm 0.006$ & ${ }^{(*)} 3 \times 10^{5} \pm 5 \times 10^{4}$ \\
\hline $\mathrm{N}$ & AM2330-451NE & - & 0.2 & $17.7 \pm 0.80$ & ${ }^{(\star)} 0.004 \pm 0.007$ & (*) $5 \times 10^{7} \pm 7 \times 10^{5}$ \\
\hline
\end{tabular}

$(\dagger) \mathcal{E} \mathcal{W}=\log (\mathcal{E W}(\mathrm{H} \alpha+[\mathrm{NII}]) \pm 0.1[\AA])$.

(*) Mass determined with $M_{B}$.

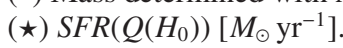

